Research Article

\title{
Adaptive Finite-Time Control on SE(3) for Spacecraft Final Proximity Maneuvers with Input Quantization
}

\author{
Sai Zhang $\mathbb{D}^{1,2}$ and Zhen Yang $\mathbb{D}^{1,2}$ \\ ${ }^{1}$ College of Aerospace Science and Engineering, National University of Defense Technology, Changsha, Hunan 410073, China \\ ${ }^{2}$ Hunan Key Laboratory of Intelligent Planning and Simulation for Aerospace Missions, Changsha, Hunan 410073, China
}

Correspondence should be addressed to Zhen Yang; yangzhen@nudt.edu.cn

Received 7 April 2021; Revised 29 July 2021; Accepted 14 August 2021; Published 15 September 2021

Academic Editor: Juan Du

Copyright (c) 2021 Sai Zhang and Zhen Yang. This is an open access article distributed under the Creative Commons Attribution License, which permits unrestricted use, distribution, and reproduction in any medium, provided the original work is properly cited.

In this paper, a bounded finite-time control strategy is developed for the final proximity maneuver of spacecraft rendezvous and docking exposed to external disturbance and input quantization. To realize the integrated control for spacecraft final proximity operation, the coupling kinematics and dynamics of attitude and position are modeled by feat of Lie group SE(3). With a view to improving the convergence rate and reducing the chattering, an adaptive finite-time controller is proposed for the error tracking system with one-step theoretical proof of stability. Meanwhile, the hysteresis logarithmic quantizer is implemented to effectively reduce the frequency of data transmission and the quantization errors are reduced under the proposed controller. The algorithms outlined above are based on an integrated model expressed by SE(3) and denoted by uniform motion states, which can simplify the design progress and improve control precision. Finally, simulations are provided to exhibit the effectiveness and advantages of the designed strategy.

\section{Introduction}

Spacecraft rendezvous and docking (RVD) technology has been successfully applied in many space missions, including space station installation and operation and deep space exploration [1-3]. Up to now, most of the RVD missions are cooperative. However, with the rapid development of space technology, noncooperative RVD is expected to serve future space activities, such as space debris removals and on-orbit services. In noncooperative RVD missions, the final proximity maneuver is severely affected by a coupled model of rotational and translational relative motions. In addition, the complicated space environment renders the maneuver control of spacecraft RVD difficult work. To overcome these two obstacles, the robust control of the spacecraft is worth more attention from the space community in future engineering missions, and some of the studies [4-7] show positive results.

In most of the existing papers, the modeling and control of relative translation and rotation in spacecraft proximity maneuvers are always supposed to be independent [5-7].
Nevertheless, the strong coupling of orbit and attitude motions makes the close-range control of noncooperative RVD extremely difficult to fulfill. Consequently, an integrated description of the relative translation channel and rotation channel is essential. Methods like dual quaternion [8-10], modified Rodrigues parameters (MRPs) [11-13], and Lie group SE(3) [14-16] were researched to build a connection between these two channels. Lie group $\operatorname{SE}(3)$ is the set of positions and orientations in the 3-D Euclidean space. Since Lie group SE(3) can provide a unique and nonsingularity description of a rigid body's motion [16], it has been applied in multibody systems $[17,18]$, heavy-duty industrial robots [19], and underwater vehicles [20]. In the field of orbit and attitude tracking control, methods using Lie group $\mathrm{SE}(3)$ have been proposed in [21-25].

Conventional linear control methods, such as PD or PID, were widely used in practical applications. These methods are deemed inapplicable to missions with complex dynamics or uncertain inertias. To promote, a great number of modified control methods, including sliding mode control 
(SMC) $[6,26]$, feedback control [27, 28], and backstepping control $[7,29]$, have been developed. Among these methods, the SMC enjoys the superiority of high robustness against uncertainty and external disturbance in a complex nonlinear system. To enhance the finite-time stability of the system, the terminal SMC (TSMC) was first proposed by Venkataraman and Gulati [30]. It is worth noting that TSMC suffers from the singularity problem, which may result in instability. Additionally, the chattering problem inherent in SMC may cause an adverse effect on actuators. To overcome these two problems, two different fast terminal sliding mode surfaces (TSMS) were proposed in $[31,32]$ to tackle the attitude tracking control problem of the spacecraft. Inspired by [31, 32] and based on the integrated model parameters, a modified nonsingular finite-time TSMS is proposed in this paper.

Besides the coupling of models and external disturbance mentioned above, the diminution of severe data transmission pressure requires further investigation from the perspective of the system's high performance and low consumption. In general, the interspacecraft communication devices mounted on the spacecraft are quite power-limited as the load of the spacecraft is limited, which results in the limited communication bandwidth [33]. Additionally, as the control modules of the spacecraft comprise digital processors, the actual control signals from the controller to the actuators must be transformed from continuous ones to discrete ones [34]. Taking these two problems into consideration, the quantizers, such as uniform quantizer, logarithmic quantizer, and hysteretic quantizer, were studied and the superiorities had been validated in [34-36]. As an improvement of the uniform quantizer, a hysteretic logarithmic quantizer (HLQ) with the property of extra quantization levels was investigated in [37], which can handle the effect of chattering. Inspired by [33-36], HLQ is implemented in this paper to reduce the communication loads and improve the control accuracy. However, the methods of $[34,36,37]$ can only ensure asymptotic convergence. As the system tends to be stable as time goes to infinity [38], asymptotic convergence cannot meet the needs of rapid, steady, and accurate control in missions with special requirements.

To the best of the authors' knowledge, few studies have been conducted from the perspective of constructing a quantized controller for spacecraft final proximity maneuver with finite-time convergence. Although Li et al. [39] proposed an adaptive controller based on sliding mode methodology and logarithmic quantizer for the RVD problem, the controller has a defect of asymptotic convergence. Accordingly, a quantized control method in conjunction with finite-time and nonsingular TSMS for spacecraft final proximity maneuver is needed.

Motivated by the above observations, it is essential but challenging to tackle the final proximity maneuver control of spacecraft RVD subject to external disturbance and quantized input. Therefore, a finite-time control algorithm is proposed in this paper based on a modified TSMS and the HLQ. The main contributions of this study can be summarized as follows:

(i) A new finite-time TSMC strategy is proposed. The finite-time stability can be analyzed in two aspects.
On the one hand, the modified TSMS with a switched function can improve the performance of system stability and avoid singularity. On the other hand, the specially designed control input in Equation (39) can provide fast finite-time stability and avoid the chattering phenomenon

(ii) By using the proposed TSMC strategy, the finitetime stability of the system can be proved directly without the upper bound value of the adaptive parameters, which is more convenient than the methods in $[5-7,32]$

(iii) The quantized control is first applied to the final proximity maneuver of spacecraft RVD modeled by Lie group $\mathrm{SE}(3)$. In comparison to the traditional time-consuming methods in [2-4], the method in this paper has sufficient merit of reducing the transmitting of tracking information and requires low communication resources

The remainder of this paper is presented as follows. The coupled model for spacecraft RVD maneuver on Lie group $\mathrm{SE}(3)$ is presented in Section 2. In Section 3, the control objective is given and a quantized TSMC algorithm is developed. Simulations are presented to validate the effectiveness of the proposed controller in Section 4, and the conclusions have been drawn in Section 5.

\section{Spacecraft Dynamics Models on SE(3)}

This section presents the kinematics and dynamics models of a spacecraft's motion around the earth and obtains the relative motion model of attitude and position tracking systems denoted by Lie group $\mathrm{SE}(3)$. Additionally, notations used in this paper are presented in this section. Three orthogonal coordinate systems are used to describe the relative motion dynamics: the Earth-centered inertial (ECI) reference frame, denoted as $\{E\}=\left\{E_{x}, E_{y}, E_{z}\right\}$ and the body-fixed frames of the chaser and the target, denoted as $\{B\}=\left\{b_{x}, b_{y}, b_{z}\right\}$ and $\left\{B_{t}\right\}=\left\{b_{t x}, b_{t y}, b_{t z}\right\}$, respectively. As shown in Figure 1. By the way, only rigid spacecraft are considered in this paper.

In this paper, $(\cdot)^{\mathrm{T}}$ expresses the transpose of a matrix and $\|\cdot\|$ denotes the Euclidean norm of a vector. For a vector $\eta=\left[\eta_{1}, \eta_{2}, \cdots, \eta_{n}\right]^{\mathrm{T}}$, it has $\operatorname{sig}(\eta)^{\beta}=\left[\operatorname{sig}\left(\eta_{1}\right)^{\beta} \cdots \operatorname{sig}\left(\eta_{n}\right)^{\beta}\right]^{\mathrm{T}}$ with $\operatorname{sig}\left(\eta_{i}\right)^{\beta}=\operatorname{sign}\left(\eta_{i}\right)\left|\eta_{i}\right|^{\beta}$.

The special group is denoted as $\operatorname{SE}(3)$ and the special orthogonal group is denoted as $\mathrm{SO}(3)$. so(3) is the Lie algebra of $\mathrm{SO}(3)$ and se(3) denotes the Lie algebra of $\mathrm{SE}(3)$.

2.1. Dynamics and Kinematics Models of a Rigid Spacecraft. In general, the kinematics model of a rigid spacecraft can be established as [4]

$$
\left\{\begin{array}{l}
\dot{b}=R v, \\
\dot{R}=R \Omega^{\times}
\end{array}\right.
$$




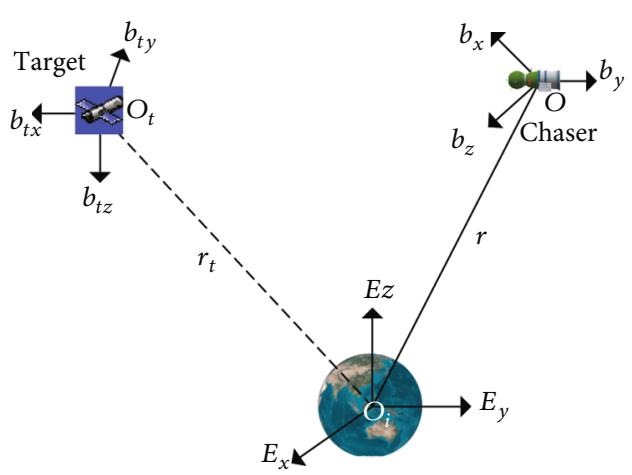

FIgURE 1: Illustration of coordinates for spacecraft proximity.

where $b=\left[\begin{array}{lll}b^{I} & b^{J} & b^{K}\end{array}\right]^{\mathrm{T}} \in \mathbb{R}^{3}$ is the inertial position of the spacecraft in $\{E\} . v \in \mathbb{R}^{3}$ and $\Omega \in \mathbb{R}^{3}$ are translational and angular velocities of the spacecraft, respectively. $R \in \mathrm{SO}(3)$ denotes the transfer matrix between the body-fixed frame and ECI. The operator $(\cdot)^{\times}: \mathbb{R}^{3} \longrightarrow \mathrm{so}(3)$ is the cross-product, which can be expressed by a $3 \times 3$ skew-symmetric matrix:

$$
a^{\times}=\left[\begin{array}{ccc}
0 & -a_{3} & a_{2} \\
a_{3} & 0 & -a_{1} \\
-a_{2} & a_{1} & 0
\end{array}\right],
$$

with $a=\left[a_{1}, a_{2}, a_{3}\right]^{\mathrm{T}}$. Let $\Phi_{c}: \operatorname{SE}(3) \times \operatorname{se}(3) \longrightarrow \mathbb{R}^{3}$ and $\tau_{c}$ $: \operatorname{SE}(3) \times \operatorname{se}(3) \longrightarrow \mathbb{R}^{3}$ denote the feedback control force and the feedback control torque, respectively. The dynamics model of a rigid spacecraft is then given as follows [4]:

$$
\left\{\begin{array}{l}
J \dot{\Omega}=J \Omega^{\times} \Omega+M^{g}(b, R)+\tau_{c}(b, R, v, \Omega)+\tau_{d}, \\
m \dot{v}=m v^{\times} \Omega+F^{g}(b, R)+m R^{T} a_{J_{2}}(b)+\Phi_{c}(b, R, v, \Omega)+\Phi_{d},
\end{array}\right.
$$

where $m$ and $J$ denote the mass and moment of inertia matrix in its body frame. $\tau_{d} \in \mathbb{R}^{3}$ and $\Phi_{d} \in \mathbb{R}^{3}$ denote the unknown moment and force acting on the chaser spacecraft, respectively. $F^{g}$ and $M^{g}$ denote the gravity force and gravity gradient moment, respectively, which are as follows:

$$
\left\{\begin{array}{l}
M^{g}=\frac{3 \mu}{\|b\|^{5}}\left(p^{\times} J p\right), \\
F^{g}=-\frac{m \mu p}{\|b\|^{3}}-\frac{3 \mu L p}{\|b\|^{5}}+\frac{15 \mu\left(p^{T} J p\right) p}{2\|b\|^{7}},
\end{array}\right.
$$

where $p=R^{\mathrm{T}} b, L=(1 / 2) \operatorname{trace}(J) I_{3}+J \cdot \mu=398600.44 \mathrm{~km}^{3} \mathrm{~s}^{-2}$ is the gravitational constant of the Earth. The perturbation caused by Earth's oblateness and $J_{2}$ in $\{E\}$ is

$$
a_{J_{2}}=\left[\begin{array}{c}
-\frac{3 \mu J_{2} R_{\oplus}^{2} b^{I}}{2\|b\|^{5}}\left(1-\frac{5\left(b^{K}\right)^{2}}{\|b\|^{3}}\right) \\
-\frac{3 \mu J_{2} R_{\oplus}^{2} b^{J}}{2\|b\|^{5}}\left(1-\frac{5\left(b^{K}\right)^{2}}{\|b\|^{3}}\right) \\
-\frac{3 \mu J_{2} R_{\oplus}^{2} b^{K}}{2\|b\|^{5}}\left(3-\frac{5\left(b^{K}\right)^{2}}{\|b\|^{3}}\right)
\end{array}\right],
$$

where $J_{2}=1.08623 \times 10^{-3}$ and $R_{\oplus}=6378 \times 10^{3} \mathrm{~km}$.

In order to tackle the problem of an integrated description of relative translation and rotation, the configuration space of rigid spacecraft motions can be denoted by the special Euclidean group $\mathrm{SE}(3)$. $\mathrm{SE}(3)$ is the semiproduct of the special orthogonal group $\mathrm{SO}(3)$ and three-dimensional position vector $\mathbb{R}^{3}$ of the spacecraft, which is expressed as $\operatorname{SE}(3)=\mathbb{R}^{3} \times \mathrm{SO}(3)$.

According to the expression in [2], the state space of the target is $\operatorname{SE}(3) \times \operatorname{se}(3) \simeq \operatorname{SE}(3) \times \mathbb{R}^{6}$ and $(b, R, v, \Omega)$ expresses its motion states. The configuration of the spacecraft on $\operatorname{SE}(3)$ can be rewritten as

$$
g=\left[\begin{array}{cc}
R & b \\
0_{1 \times 3} & 1
\end{array}\right] \in \mathrm{SE}(3)
$$

where $0_{1 \times 3}$ means a $1 \times 3$ null matrix.

Here, it defines the angular velocity $\Omega$ and translational velocity $v$ in a unified form as $\xi=\left[\begin{array}{ll}\Omega^{\mathrm{T}} & v^{\mathrm{T}}\end{array}\right]^{\mathrm{T}} \in \mathbb{R}^{6}$. Thus, the kinematics model in Equation (1) is reconstructed as

$$
\dot{g}=g \xi^{\vee}
$$

where $\xi^{\vee}=\left[\begin{array}{cc}\Omega^{\times} & v \\ 0_{1 \times 3} & 0\end{array}\right] \in \operatorname{se}(3)$ is the velocity mapping. The denotation $\varphi^{g}$ is introduced to express the vector of gravitational force and moment and $\Xi$ denotes the mass and inertia of spacecraft, respectively. $\varphi^{g}$ and $\Xi$ are defined as

$$
\begin{gathered}
\varphi^{g}=\left[\begin{array}{c}
M^{g} \\
F^{g}+m R^{\mathrm{T}} a_{J_{2}}
\end{array}\right] \in \mathbb{R}^{6}, \\
\Xi=\left[\begin{array}{cc}
J & 0_{3 \times 3} \\
0_{3 \times 3} & m I_{3}
\end{array}\right] \in \mathbb{R}^{6 \times 6} .
\end{gathered}
$$

Similar to the definition of adjoint action between a Lie group and its corresponding Lie algebra, the adjoint operator and coadjoint representation of $\mathrm{SE}(3)$ on $\mathrm{se}(3)$ can be given by [21]: 


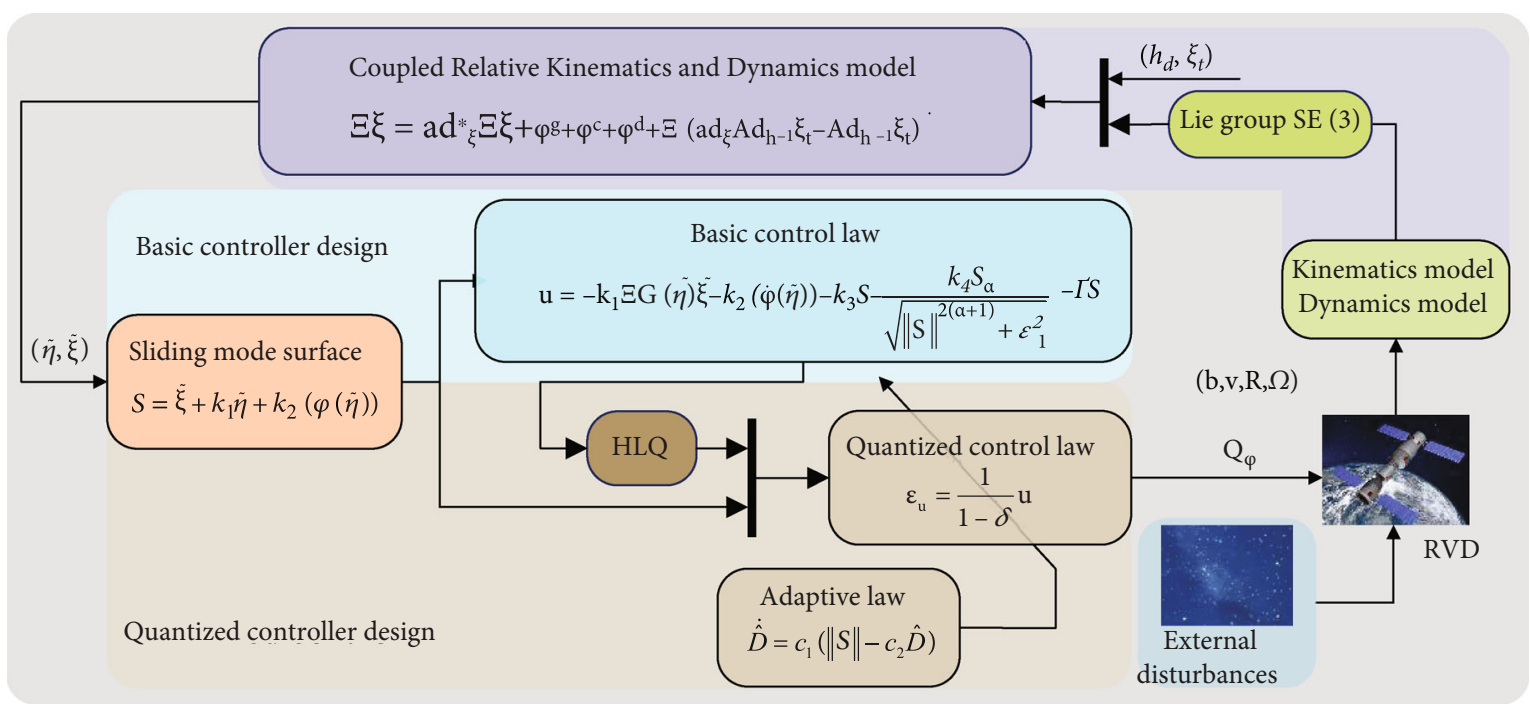

FIGURE 2: Block diagram of the control architecture for spacecraft rendezvous motion.

$$
\operatorname{Ad}_{g}=\left[\begin{array}{cc}
R & 0_{3 \times 3} \\
b^{\times} R & R
\end{array}\right] \in \mathbb{R}^{6 \times 6} \text { s.t. } \operatorname{Ad}_{g} X^{\wedge}=\left(g X g^{-1}\right)^{\wedge},
$$

where $(\cdot)^{\wedge}: \operatorname{se}(3) \longrightarrow \mathbb{R}^{6}$ is the inverse of $(\cdot)^{\vee}: \mathbb{R}^{6}$ $\longrightarrow \mathrm{se}(3)$. In matrix form, the adjoint representation of se (3) is expressed in (10a) and the coadjoint representation is given in (10b).

$$
\begin{gathered}
\operatorname{ad}_{\xi}=\left[\begin{array}{cc}
\Omega^{\times} & 0_{3 \times 3} \\
v^{\times} & \Omega^{\times}
\end{array}\right] \in \mathbb{R}^{6 \times 6}, \\
\operatorname{ad}_{\xi}^{*}=\left(\operatorname{ad}_{\xi}\right)^{\mathrm{T}}=\left[\begin{array}{cc}
-\Omega^{\times} & -v^{\times} \\
0_{3 \times 3} & -\Omega^{\times}
\end{array}\right] .
\end{gathered}
$$

In virtue of the coadjoint operator, the dynamics model in Equation (3) can be expressed in a compact form [21]:

$$
\Xi \dot{\xi}=\operatorname{ad}_{\xi}^{*} \Xi \xi+\varphi^{g}+\varphi^{c}+\varphi^{d}
$$

where $\varphi^{c}=\left[\begin{array}{c}\tau_{c} \\ \Phi_{c}\end{array}\right]$ and $\varphi^{d}=\left[\begin{array}{c}\tau_{d} \\ \Phi_{d}\end{array}\right]$. Specifically, $\varphi^{c} \in \mathbb{R}^{6}$ denotes the control input and $\varphi^{d} \in \mathbb{R}^{6}$ denotes the external disturbance.

Similarly, denote $\mathbf{R}_{t} \in \mathrm{SO}(3)$ to be the transfer matrix between $\left\{B_{t}\right\}$ and $\{E\}$. The inertial position, translational velocity, and the angular velocity of the target can be denoted as $b_{t}=\left[\begin{array}{lll}b_{t}^{I} & b_{t}^{J} & b_{t}^{K}\end{array}\right]^{\mathrm{T}} \in \mathbb{R}^{3}, v_{t} \in \mathbb{R}^{3}$ and $\Omega_{t} \in \mathbb{R}^{3}$, respectively. $m_{t}$ and $J_{t}$ denote the target's mass and moment of inertia matrix in its body frame. The configuration of the target on $\operatorname{SE}(3)$ can be given as follows:

$$
g_{t}=\left[\begin{array}{cc}
R_{t} & b_{t} \\
0_{1 \times 3} & 1
\end{array}\right] \in \mathrm{SE}(3)
$$

The dynamics model of the target on Lie Group $\operatorname{SE}(3)$ is

$$
\Xi_{t} \dot{\xi}_{t}=\operatorname{ad}_{\xi_{t}}^{*} \Xi_{t} \xi_{t}+\varphi_{t}^{g}
$$

with $\xi_{t}=\left[\begin{array}{cc}\Omega_{t}^{\mathrm{T}} & v_{t}^{\mathrm{T}}\end{array}\right]^{\mathrm{T}} \in \mathbb{R}^{6}$ and $\Xi_{t}=\left[\begin{array}{cc}J_{t} & 0_{3 \times 3} \\ 0_{3 \times 3} & m_{t} I_{3}\end{array}\right]$. The inertial state is $g_{t}\left(t_{0}\right)=\xi_{t}\left(t_{0}\right)$ at time $t_{0}$. At the meantime, the state trajectory of the target for $t \geq t_{0}$ can be generated by using Equations (12) and (13).

Remark 1. The kinematics and dynamics for a normal rigid spacecraft are defined by the equations presented previously (see Equation (7) and (11)). It needs to emphasize that the target spacecraft is assumed to maneuver in the gravity field, and no external disturbance or control input is acting on the target. It is important not to confuse the chaser and the target.

\subsection{Relative Dynamical Model for Spacecraft Proximity} Operation. It defines the relative configuration and desired relative configuration between the chaser and the target to be $h \in \operatorname{SE}(3)$ and $h_{f} \in \operatorname{SE}(3)$. According to the tracking trajectory created by Equations (6) and (12), the desired states of the chaser spacecraft are as follows:

$$
\begin{gathered}
g_{d}=g_{t} h_{f}, \\
\xi_{d}=\operatorname{Ad}_{\mathbf{h}_{f}^{-1}} \xi_{t} \text { for } t \geq 0 .
\end{gathered}
$$

And the configuration error between the chaser and target is

$$
h=g_{t}^{-1} g \text { for } t \geq 0,
$$




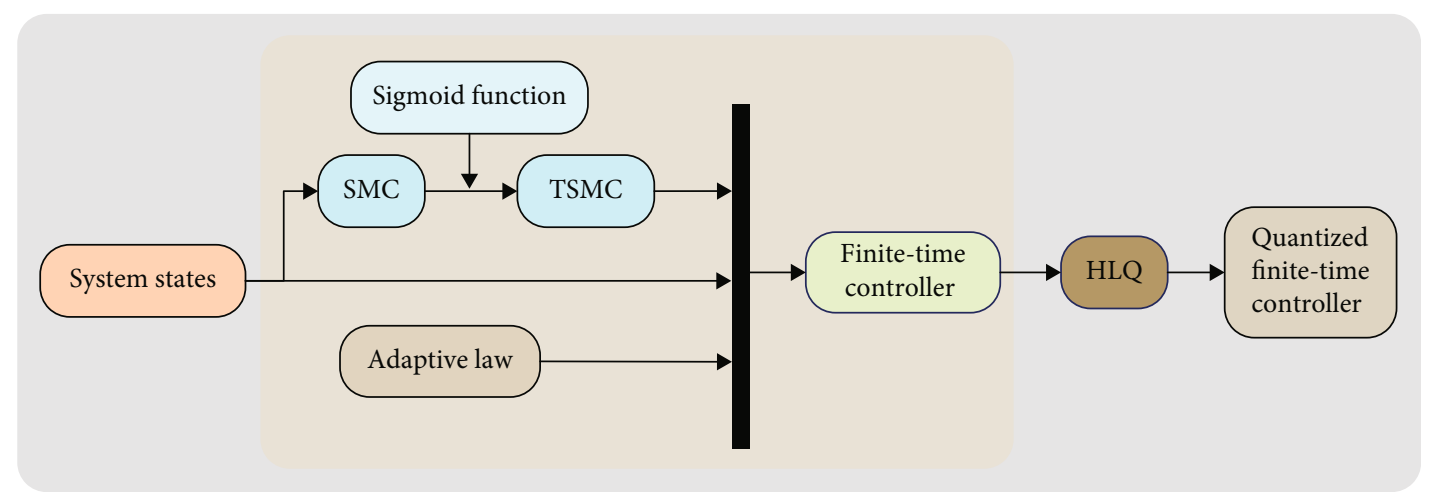

FIgURE 3: Block diagram of the controller design process.

where $\operatorname{Ad}_{\mathbf{h}_{f}^{-1}}$ denotes the adjoint action of $h_{f}$. In view of the logarithm map, the configuration error can be expressed in exponential coordinate, that is

$$
\tilde{\eta}^{\vee}=\log _{\mathrm{SE}(3)}\left(h_{f}^{-1} h\right)=\log _{\mathrm{SE}(3)}\left(g_{d}^{-1} g\right)
$$

where $\log _{\mathrm{SE}(3)}: \mathrm{SE}(3) \longrightarrow \mathrm{se}(3)$ is the logarithm map $(\operatorname{logm})$. Thus, the relative configuration between the actual and desired configuration can be defined by the exponential coordinate vector $\tilde{\eta}$ of the chaser, which is

$$
\tilde{\eta}=\left[\begin{array}{ll}
\tilde{\Theta} & \tilde{\beta}
\end{array}\right]^{\mathrm{T}} \in \mathbb{R}^{6}
$$

where $\tilde{\Theta} \in \mathbb{R}^{3}$ and $\tilde{\beta} \in \mathbb{R}^{3}$ represent the attitude tracking error and position tracking error, respectively.

Remark 2. The exponential map $\exp _{\mathrm{SE}(3)}$ from se(3) to $\mathrm{SE}(3)$ is a surjective map and a local diffeomorphism. $\exp _{\mathrm{SE}(3)}$ is bijective when $\|\Theta\| \leq \pi$. The inverse expression of $\exp _{\mathrm{SE}(3)}$ is a logarithm map denoted as $\log _{\mathrm{SE}(3)}$. The standard computations of $\exp _{\mathrm{SE}(3)}$ and $\log _{\mathrm{SE}(3)}$ are presented in [40]. According to Equations (14)-(17), one can conclude that $\tilde{\eta}^{\vee}=\left[\begin{array}{c}\tilde{\Theta} \\ \tilde{\beta}\end{array}\right]^{\vee}=\log _{\operatorname{SE}(3)}\left(h_{f}^{-1} h\right)=\log _{\operatorname{SE}(3)}\left(\mathrm{h}_{\mathrm{e}}\right)$ and $\left.\exp _{\mathrm{SE}(3)}\left(\begin{array}{c}\tilde{\Theta} \\ \tilde{\beta}\end{array}\right]^{\vee}\right)=h_{e}=\left[\begin{array}{cc}R_{e} & b_{e} \\ 0_{1 \times 3} & 1\end{array}\right]$. The solution process can be found in [40]. Apart from that, for any initial relative attitude and any initial relative principal rotation axis, the norm of $R_{e}$ is 1 , which has been proven in Appendix A. As a result, the exponential coordinate vector $(\tilde{\Theta}, \tilde{\beta})$ has a strict relationship with $\left(R_{e}, b_{e}\right)$ and can represent the attitude tracking error and position tracking error.

With the aid of Equations (7) and (12), the relative velocity is expressed as

$$
\tilde{\xi}=\xi-\operatorname{Ad}_{h^{-1}} \xi_{t}=\xi-\operatorname{Ad}_{h_{f}^{-1}} \xi_{d}
$$

where $\tilde{\xi}$ represents the relative velocity of the chaser with respect to the target. The first-order derivative of $\tilde{\xi}$ is

$$
\dot{\tilde{\xi}}=\dot{\xi}+\operatorname{ad}_{\xi} \operatorname{Ad}_{h^{-1}} \xi_{t}-\operatorname{Ad}_{h^{-1}} \dot{\xi}_{t}
$$

It defines that $h_{0}=h\left(t_{0}\right), \tilde{\eta}_{0}=\eta\left(t_{0}\right), \xi_{0}=\xi\left(t_{0}\right)$, and $\tilde{\xi}_{0}=$ $\tilde{\xi}\left(t_{0}\right)$ to be the initial states at time $t_{0}$, which are assumed to be known as long as the initial states of the chaser spacecraft are known. According to [21], the kinematics model of exponential coordinates is

$$
\dot{\tilde{\eta}}=G(\tilde{\eta}) \tilde{\xi}
$$

where $G(\tilde{\eta})$ is a block-triangular matrix. Given that $\eta=$ $\left[\begin{array}{ll}\Theta^{\mathrm{T}} & \beta^{\mathrm{T}}\end{array}\right]^{\mathrm{T}} \in \mathbb{R}^{6}$ denotes the actual configuration, the function $G(\eta)$ can be defined as

$$
G(\eta)=\left[\begin{array}{cc}
A(\Theta) & 0_{3 \times 3} \\
T(\Theta, \beta) & A(\Theta)
\end{array}\right]
$$

where $A(\Theta)=I_{3}+(1 / 2) \Theta^{\times}+\left(\left(1 / \theta^{2}\right)-((1+\cos \theta) / 2 \theta \sin \theta)\right)$ $\left(\Theta^{\times}\right)^{2}, \quad S(\Theta)=I_{3}+\left((1-\cos \theta) / \theta^{2}\right) \Theta^{\times}+()\left(\theta-\sin \theta / \theta^{3}\right)$ $\left(\Theta^{\times}\right)^{2}$, and $T(\Theta, \beta)=\left(1 / \theta^{2}-1+\cos \theta / 2 \theta \sin \theta\right)\left(\Theta \beta^{\mathrm{T}}+\right.$ $\left.\left(\Theta^{\mathrm{T}} \beta\right) A(\Theta)\right)+1 / 2(S(\Theta) \beta)^{\times} A(\Theta)+((1+\cos \theta)(\theta+\sin \theta) / 2$ $\left.\theta^{3} \sin ^{2} \theta-2 / \theta^{4}\right) \Theta^{\mathrm{T}} \beta \Theta \Theta^{\mathrm{T}}-\left((1+\cos \theta)(\theta-\sin \theta) / 2 \theta \sin ^{2} \theta\right)$ $S(\Theta) \beta \Theta^{\mathrm{T}}$ with $\theta=\|\Theta\|$ and $R(\Theta)=\exp _{S O(3)}(\Theta)=I_{3}+(\sin$ $\theta / \theta) \Theta^{\times}+\left((1-\cos \theta) / \theta^{2}\right)\left(\Theta^{\times}\right)^{2}$.

According to [21], $G(\eta)$ satisfies this relation:

$$
G(\eta) \eta=\eta
$$

Equation (22) has been proved in [21]. Thus, $G(\eta)$ is a positive definite matrix when $\eta \neq 0$ and $G(\eta)=I$ when $\eta=$ 0 .

Substituting Equations (11) and (13) into Equation (19) leads to the first-order derivative of relative acceleration:

$$
\Xi \dot{\tilde{\xi}}=\operatorname{ad}_{\xi}^{*} \Xi \xi+\varphi^{g}+\varphi^{c}+\varphi^{d}+\Xi\left(\operatorname{ad}_{\xi} \operatorname{Ad}_{h^{-1}} \xi_{t}-\operatorname{Ad}_{h^{-1}} \dot{\xi}_{t}\right)
$$

By combining the relative kinematics expressed by exponential coordinate in (20) and the relative acceleration 
equation in Equation (23), the coupled error tracking system of spacecraft final proximity maneuvers is established.

\section{Quantized Finite-Time TSMC Scheme Design and Stability Analysis}

In this section, a finite-time controller is proposed for spacecraft RVD considering external disturbance and input quantization. Firstly, it gives the preliminaries. To better show the aim and design process of this paper, the schematic diagram and the control objective are given. Finally, in conjunction with adaptive algorithms and a switched TSMS, a quantized finite-time TSMC algorithm is proposed. Via the proposed control scheme, the finite-time stability of the system can be proved directly.

3.1. Preliminaries. A quantizer refers to the existing results in [37]; the HLQ is introduced here to replace the traditional time-consuming methods:

$$
Q(u)= \begin{cases}u_{i} \operatorname{sgn}(u), & \frac{u_{i}}{1+\delta}<|u| \leq u_{i}, \dot{u}<0 \text { or } \\ & u_{i}<|u| \leq \frac{u_{i}}{1-\delta}, \dot{u}>0, \\ u_{i}(1+\delta) \operatorname{sgn}(u), & u_{i}<|u| \leq \frac{u_{i}}{1-\delta}, \dot{u}<0 \text { or } \\ & \frac{u_{i}}{1-\delta}<|u| \leq \frac{u_{i}(1+\delta)}{1-\delta}, \dot{u}>0, \\ 0, & 0 \leq|u|<\frac{u_{\min }}{1+\delta}, \dot{u}<0 \text { or } \\ Q\left(u\left(t^{-}\right)\right), & \frac{u_{\min }}{1+\delta}<u \leq u_{\min }, \dot{u}>0, \\ & \dot{u}=0,\end{cases}
$$

where $u_{i}=\rho^{1-i} u_{\min }(i=1,2, \cdots), u_{\min }>0$ denotes the range of the hysteresis zone for $Q(u)$, and $\delta$ determines the transmitting rate of the communication channel and satisfies $\delta=(1-\rho) /(1+\rho)$ with $0<\rho<1$. Obviously, $Q(u)$ is in the set of $U=\left\{0, \pm u_{i}, \pm u_{i}(1+\delta)\right\}$.

The HLQ can be decomposed into a continuous part and a discontinuous part [41], which is

$$
Q(u)=\kappa(u) u+E(u)
$$

where $\quad \kappa(u)=\left\{\begin{array}{ll}Q(u) / u, & Q(u) \neq 0 \\ 1, & Q(u)=0\end{array}\right.$ and $\quad E(u)=$ $\left\{\begin{array}{ll}0, & Q(u) \neq 0 \\ -u, & Q(u)=0\end{array}\right.$.

Remark 3. The control signals transmitted to the actuators are quantized in the system with an HLQ. The quantizer here can convert continuous signals into discrete signals numerically, which can reduce the burden of information transmission. In this study, $u_{\min }$ and $\delta$ of each dimension of control input are supposed to be the same.
TABLE 1: Initial orbital elements of the target $[1,43]$.

\begin{tabular}{lc}
\hline Orbital element & Value \\
\hline Semimajor axis $a(\mathrm{~km})$ & 26628 \\
Eccentricity $e$ & 0.7417 \\
Inclination $i(\mathrm{deg})$ & 63.4 \\
RAAN $\Omega(\mathrm{deg})$ & 0 \\
Argument of perigee $\omega(\mathrm{deg})$ & 270 \\
The anomaly $f(\mathrm{deg})$ & 120 \\
\hline
\end{tabular}

To promote the design, the following lemmas and assumptions are essential for the establishment of the control algorithm.

Lemma 4 (see [41]). It is easy to find that $\kappa(u)$ is continuous and $E(u)$ is discontinuous; thus, the following inequality holds:

$$
\left\{\begin{array}{l}
1-\delta \leq \kappa(u) \leq 1+\delta \\
E(u) \leq u_{\min }
\end{array}\right.
$$

where $\delta$ and $u_{\min }$ are design parameters of $H L Q$.

Lemma 5. For any $\varepsilon>0$ and $x \in \mathbb{R}$, the following inequality holds:

$$
0 \leq|x|-\frac{|x|^{2}}{\sqrt{x^{2}+\varepsilon^{2}}} \leq|x|-\frac{|x|^{2}}{|x|+\varepsilon}<\varepsilon
$$

Lemma 6. For any $1>b>0$ and $x_{i} \in \mathbb{R}(i=1, \cdots, n)$, the following inequality holds:

$$
\left(\left|x_{1}\right|+\cdots+\left|x_{n}\right|\right)^{b} \leq\left|x_{1}\right|^{b}+\cdots+\left|x_{n}\right|^{b} .
$$

Lemma 7. Based on Equations (27) and (28), the following inequation can be obtained for a vector $x=\left[\begin{array}{llll}x_{1} & x_{2} & \cdots & x_{n}\end{array}\right]^{T}$ :

$$
-\frac{x^{T} x^{2 \alpha+1}}{\sqrt{\|x\|^{2(\alpha+1)}+\varepsilon^{2}}} \leq-\|x\|^{\alpha+1}+\varepsilon,
$$

where $x^{2 \alpha+1}=\left[\begin{array}{llll}x_{1}^{2 \alpha+1} & x_{2}^{2 \alpha+1} & \cdots & x_{n}^{2 \alpha+1}\end{array}\right]^{T}$, and $\alpha$ is defined as

$$
\alpha=-\frac{m}{2 n+1}
$$

where $0<2 m<2 n+1$ with $m, n \in \mathbb{N}_{+}$. It is obvious that $-1 / 2$ $<\alpha<0$.

Lemma 8. According to Young's inequality, for a constant $\mu$, if $\widehat{\mu}+\tilde{\mu}=\mu$ holds, one has

$$
\widehat{\mu} \tilde{\mu} \leq-\frac{1}{2} \tilde{\mu}^{2}+\frac{1}{2} \mu^{2} .
$$


TABLE 2: Initial states of the chaser relative to the target $[1,43]$.

\begin{tabular}{|c|c|}
\hline Initial relative states & Value \\
\hline Target's angular velocity ( $\mathrm{rad} / \mathrm{s})$ & {$\left[\begin{array}{lll}0 & 0 & 0.0011\end{array}\right]^{\mathrm{T}}$} \\
\hline Initial relative position $(\mathrm{m})$ & {$\left[\begin{array}{lll}15 & 15 & 15\end{array}\right]^{\mathrm{T}}$} \\
\hline Initial relative velocity $(\mathrm{m} / \mathrm{s})$ & {$\left[\begin{array}{lll}-0.051 & -0.247 & -0.075\end{array}\right]^{\mathrm{T}}$} \\
\hline Initial relative attitude (rad) & $2 \pi / 3$ \\
\hline Initial relative principal rotation axis & {$\left[\begin{array}{lll}-2 & -2 & -3\end{array}\right]^{\mathrm{T}}$} \\
\hline Initial relative angular velocity ( $\mathrm{rad} / \mathrm{s})$ & {$\left[\begin{array}{lll}0.009 & 5.98 & -9.31\end{array}\right]^{\mathrm{T}} \times 10^{-4}$} \\
\hline
\end{tabular}

Lemma 9 (see [42]). For the system in Equation (23), if a Lyapunov function $V$ exists and satisfies

$$
\dot{V} \leq-\alpha V^{\gamma}+\sigma
$$

with $1>\gamma>0, \alpha>0$, and $0 \leq \sigma<\infty$, then the trajectory of Equation (23) is practical finite-time stable.

Lemmas 5 and 6 are easy to be proved by virtue of mathematical expansion. The proof of Lemma 7 is given in Appendix B.

Remark 10. There is a condition: $\alpha=-m /(2 n+1)$ with $0<$ $2 m<2 n+1$. Hence, for any constant $x \in \mathbb{R}, x^{\alpha}$, and $x^{1+2 \alpha}$ will make sense. By the way, the value of $\alpha$ can ensure finite-time convergence for system states, which will be illustrated in the following text.

Assumption 11. The inertial matrix $J$ and mass $m_{p}$ of the chaser spacecraft are known. The inertial matrix $J_{t}$ and mass $m_{t}$ of the target are unknown. Both $J$ and $J_{t}$ are constant positive-definite symmetric matrices. $m_{p}$ and $m_{t}$ are bounded constants.

Assumption 12. All the disturbances are unknown but bounded with an upper bound. That is, $\left\|\varphi^{d}\right\| \leq D$ with $D$ being an uncertain positive constant.

Remark 13. With the help of onboard devices equipped on spacecraft, the information of the chaser can be interactive. Thus, Assumption 11 is reasonable. As for Assumption 12, it is believed that environmental interference is limited and the unknown disturbance can be strictly dominated by control inputs.

For the control objectives, in this paper, we denote to designing a finite-time TSMC scheme for spacecraft RVD subject to external disturbance and input quantization. The objective is to design the control input command $\varphi^{c}$ for the error system in Equation (23) under Assumptions 11 and 12 , such that all closed-loop signals are guaranteed to be bounded and the global system is finite-time stable. For a better illustration, the architecture of the controller synthesis is shown in Figure 2.
TABLE 3: Desired states of the chaser relative to the target $[1,43]$.

\begin{tabular}{lcc}
\hline Desired relative states & \multicolumn{2}{c}{ Value } \\
\hline Desired relative position $(\mathrm{m})$ & {$\left[\begin{array}{ccc}5 & 0 & 0\end{array}\right]^{\mathrm{T}}$} \\
Desired relative velocity $(\mathrm{m} / \mathrm{s})$ & {$\left[\begin{array}{ccc}0 & 0 & 0\end{array}\right]^{\mathrm{T}}$} \\
Desired relative attitude $(\mathrm{rad})$ & 0 & \\
Initial relative angular velocity $(\mathrm{rad} / \mathrm{s})$ & {$\left[\begin{array}{lll}0 & 0 & 0\end{array}\right]^{\mathrm{T}}$} \\
\hline
\end{tabular}

3.2. Quantized Finite-Time TSMC Design. Inspired by [37], the actual control input is the quantized control torque, and as a result, Equation (23) is rewritten as

$$
\Xi \dot{\tilde{\xi}}=\operatorname{ad}_{\xi}^{*} \Xi \xi+\varphi^{g}+Q_{\varphi}+\varphi^{d}+\Xi\left(\operatorname{ad}_{\xi} \operatorname{Ad}_{h^{-1}} \xi_{t}-A d_{h^{-1}} \dot{\xi}_{t}\right),
$$

where $Q_{\varphi}=\left[\begin{array}{llllll}Q_{\phi}^{1} & Q_{\phi}^{2} & Q_{\phi}^{3} & Q_{\phi}^{4} & Q_{\phi}^{5} & Q_{\phi}^{6}\end{array}\right] \in \mathbb{R}^{6}$ is the quantized value of the designed control input $\varepsilon_{u}$. The quantized input $Q_{\varphi}$ can be written as

$$
Q_{\varphi}=\kappa(u) \boldsymbol{\varepsilon}_{u}+E(u) I_{1}
$$

with $I_{1}=\left[\begin{array}{llllll}1 & 1 & 1 & 1 & 1 & 1\end{array}\right]^{\mathrm{T}} . \kappa(u)$ and $E(u)$ satisfy the inequality in Equation (26) with $\delta$ and $u_{\min }$ being design parameters.

By using the Sigmoid function, a modified switched TSMS $S=\left[\begin{array}{llllll}S_{1} & S_{2} & S_{3} & S_{4} & S_{5} & S_{6}\end{array}\right]^{\mathrm{T}} \in \mathbb{R}^{6}$ is designed as follows:

$$
S=\tilde{\xi}+k_{1} \tilde{\eta}+k_{2}(\varphi(\tilde{\eta}))
$$

where $k_{1}=\operatorname{diag}\left(k_{11}, k_{12}, k_{13}, k_{14}, k_{15}, k_{16}\right)$ and $k_{2}=\operatorname{diag}\left(k_{21}\right.$ $\left., k_{22}, k_{23}, k_{24}, k_{25}, k_{26}\right)$ with $k_{1 i}(i=1,2,3,4,5,6)$ and $k_{2 i}(i=$ $1,2,3,4,5,6)$ being positive constants. In particular, $\varphi(\tilde{\eta})$ $=\left[\begin{array}{llllll}\varphi\left(\tilde{\eta}_{1}\right) & \varphi\left(\tilde{\eta}_{2}\right) & \varphi\left(\tilde{\eta}_{3}\right) & \varphi\left(\tilde{\eta}_{4}\right) & \varphi\left(\tilde{\eta}_{5}\right) & \varphi\left(\tilde{\eta}_{6}\right)\end{array}\right]^{\mathrm{T}} \quad$ is $\quad$ a piecewise smooth function and $\varphi\left(\tilde{\eta}_{i}\right)$ is defined as

$$
\varphi\left(\tilde{\eta}_{i}\right)= \begin{cases}\operatorname{sig}\left(\tilde{\eta}_{i}\right)^{\beta}, & \left|\tilde{\eta}_{i}\right|>\vartheta \\ \frac{2 \vartheta}{\pi} \sin \left(\frac{\pi}{2 \vartheta} \tilde{\eta}_{i}\right)+\beta \vartheta^{\beta-1} \tilde{\eta}_{i}, & \text { otherwise }\end{cases}
$$


TABLE 4: Three different kinds of SMC schemes.

\begin{tabular}{|c|c|c|}
\hline Component & SMS & Control inputs \\
\hline$S_{\text {proposed }}$ & $S=\tilde{\xi}+k_{1} \tilde{\eta}+k_{2}(\varphi(\tilde{\eta}))$ & $\begin{array}{c}u=-k_{1} \Xi G(\tilde{\eta}) \tilde{\xi}-k_{2}(\dot{\varphi}(\tilde{\eta}))-k_{3} S-k_{4} S_{\alpha} / \sqrt{\|S\|^{2(\alpha+1)}+\varepsilon_{1}^{2}}-\Gamma S \text { with } \Gamma= \\
\left(\left\|\Lambda_{\varphi}\right\|^{2} / \sqrt{\left\|\Lambda_{\varphi}\right\|^{2}\|S\|^{2}+\varepsilon_{1}^{2}}\right)+\left(D \wedge^{2} / \sqrt{D \wedge^{2}\|S\|^{2}+\varepsilon_{1}^{2}}\right)\end{array}$ \\
\hline$S_{\text {sig }}$ & $S_{s}=\tilde{\xi}+b_{1} \tilde{\eta}+b_{2} \operatorname{sig}(\tilde{\eta})^{\zeta}$ & $\begin{array}{c}u_{s}=-b_{1} \Xi G(\tilde{\eta}) \tilde{\xi}-b_{2} \zeta \operatorname{dsig}(\tilde{\eta})-b_{3} S-\left(\left\|\Lambda_{\varphi}\right\|+D\right) \operatorname{sig}\left(S_{\alpha}\right)^{\zeta}-b_{4} \operatorname{sig}\left(S_{\alpha}\right)^{\zeta} \text { with } \operatorname{dsig}(\tilde{\eta})= \\
{\left[\begin{array}{lll}\left|\tilde{\eta}_{1}\right|^{\zeta-1} \dot{\tilde{\eta}}_{1} & \cdots & \left|\tilde{\eta}_{6}\right|^{\zeta-1} \dot{\tilde{\eta}}_{6}\end{array}\right]^{\mathrm{T}}}\end{array}$ \\
\hline$S_{\text {tanh }}$ & $S_{t}=\tilde{\xi}+l_{1} \tilde{\eta}+l_{2} \tanh (\tilde{\eta})$ & $u_{t}=-l_{1} \Xi G(\tilde{\eta}) \tilde{\xi}-l_{2} \dot{\tilde{\eta}}-l_{3} S_{t}-l_{4} \tanh \left(S_{t}\right)-\left(\left\|\Lambda_{\varphi}\right\|+D\right) \tanh \left(S_{t}\right)$ \\
\hline
\end{tabular}

TABLE 5: Gains of the three control schemes.

\begin{tabular}{|c|c|}
\hline Component & Value \\
\hline SMS gains & $\begin{array}{c}k_{1}=0.1 \times \operatorname{diag}\{0.52,0.52,0.52,3.6,3.6,3.6\}, \\
k_{2}=10^{-3} \times \operatorname{diag}\{15,15,15,15,15,15\} . \\
b_{1}=10^{-2} \times \operatorname{diag}\{3.6,3.6,3.6,12.5,12.5,12.5\}, \\
b_{2}=10^{-2} \times \operatorname{diag}\{1.6,1.6,1.6,15,15,15\} . \\
l_{1}=10^{-2} \times \operatorname{diag}\{2.2,2.2,2.2,27,27,27\}, l_{2}=10^{-2} \times \operatorname{diag}\{4,4,4,2,2,2\} .\end{array}$ \\
\hline Control gains & $\begin{array}{c}k_{3}=\operatorname{diag}\{6.6,6.6,6.6,6,6,6\}, k_{4}=\operatorname{diag}\{10,10,10,6,6,6\} . \\
b_{3}=\operatorname{diag}\{11.5,11.5,11.5,5.8,5.8,5.8\}, b_{4}=\operatorname{diag}\{11,11,11,10,10,10\} . \\
l_{1}=\operatorname{diag}\{13.5,13.5,13.5,7,7,7\}, l_{2}=\operatorname{diag}\{12,12,12,15,15,15\} .\end{array}$ \\
\hline Adaptive gains & $c_{1}=0.12, c_{2}=4$ \\
\hline Other input parameters & $\alpha=-1 / 9, \beta=\kappa=0.6, \varepsilon_{1}=0.1, \zeta=0.6$ \\
\hline
\end{tabular}

where $0<\beta<1$, and $\vartheta$ is a small positive constant, which is defined as

$$
\vartheta=\left(\frac{\pi}{2}(1-\kappa)\right)^{1 /(1-\kappa)}
$$

with $0<\kappa<1$. The derivative of $\varphi(\tilde{\eta})$ is $\dot{\varphi}(\tilde{\eta})=$

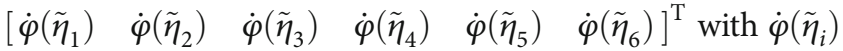
being

$$
\dot{\varphi}\left(\tilde{\eta}_{i}\right)= \begin{cases}\beta\left|\tilde{\eta}_{i}\right|^{\beta-1} \dot{\tilde{\eta}}_{i}, & \left|\tilde{\eta}_{i}\right|>\vartheta \\ \cos \left(\frac{\pi}{2 \vartheta} \tilde{\eta}_{i}\right) \dot{\tilde{\eta}}_{i}+\beta \vartheta^{\beta-1} \dot{\tilde{\eta}}_{i}, & \text { otherwise. }\end{cases}
$$

The actual control input $Q_{\varphi}$ is determined by $\boldsymbol{\varepsilon}_{u}$, which is designed as

$$
\begin{gathered}
\boldsymbol{\varepsilon}_{u}=\frac{1}{1-\delta} u \\
u=-k_{1} \Xi G(\tilde{\eta}) \tilde{\xi}-k_{2}(\dot{\varphi}(\tilde{\eta}))-k_{3} S-\frac{k_{4} S_{\alpha}}{\sqrt{\|S\|^{2(\alpha+1)}+\varepsilon_{1}^{2}}}-\Gamma S
\end{gathered}
$$

with $S_{\alpha}=\left[\begin{array}{llllll}S_{1}^{2 \alpha+1} & S_{2}^{2 \alpha+1} & S_{3}^{2 \alpha+1} & S_{4}^{2 \alpha+1} & S_{5}^{2 \alpha+1} & S_{6}^{2 \alpha+1}\end{array}\right]^{\mathrm{T}} \cdot k_{3}$ and $k_{4}$ are diagonal matrixes denoted as $k_{3}=\operatorname{diag}\left(k_{31}, k_{32}\right.$, $\left.k_{33}, k_{34}, k_{35}, k_{36}\right)$ and $k_{4}=\operatorname{diag}\left(k_{41}, k_{42}, k_{43}, k_{44}, k_{45}, k_{46}\right)$ with $k_{3 i}(i=1,2,3,4,5,6)$ and $k_{4 i}(i=1,2,3,4,5,6)$ being positive constants, which are humanly designed and will not change. $\Gamma=\left(\left\|\Lambda_{\varphi}\right\|^{2} / \sqrt{\left\|\Lambda_{\varphi}\right\|^{2}\|S\|^{2}+\varepsilon_{1}^{2}}\right)+\left(D \wedge^{2} / \sqrt{D \wedge^{2}\|S\|^{2}+\varepsilon_{1}^{2}}\right)+$ $\left(u^{2} / \sqrt{D \wedge^{2}\|S\|^{2}+\varepsilon_{1}^{2}}\right)$ is a time-varying state variable with $\Lambda_{\varphi}$ $=\operatorname{ad}_{\xi}^{*} \Xi \xi+\varphi^{g}+\Xi\left(\operatorname{ad}_{\xi} \operatorname{Ad}_{h^{-1}} \xi_{t}-\operatorname{Ad}_{h^{-1}} \dot{\xi}_{t}\right) . u$ is a constant satisfying $u_{\min } \leq u$. $\widehat{D}$ is the estimation of $D$.

The adaptive law of $D$ is designed as follows:

$$
\dot{\widehat{D}}=c_{1}\left(\|S\|-c_{2} \widehat{D}\right)
$$

where $c_{1}$ and $c_{2}$ are positive constants. The estimated error of $D$ is defined as

$$
\tilde{D}=D-\widehat{D} \text {. }
$$

Remark 14. Three components comprise the quantized finite-time controller: the adaptive law, the finite-time input, and the quantizer. The finite-time input is made up of a switched TSMS (Equations (35)- (37)) and a modified finite-time convergent term (Equation (39b)). Combined with the adaptive law, the basic controller is obtained. Additionally, an HLQ is implemented to transfer the control signal, and, meanwhile, the basic controller is then altered by 


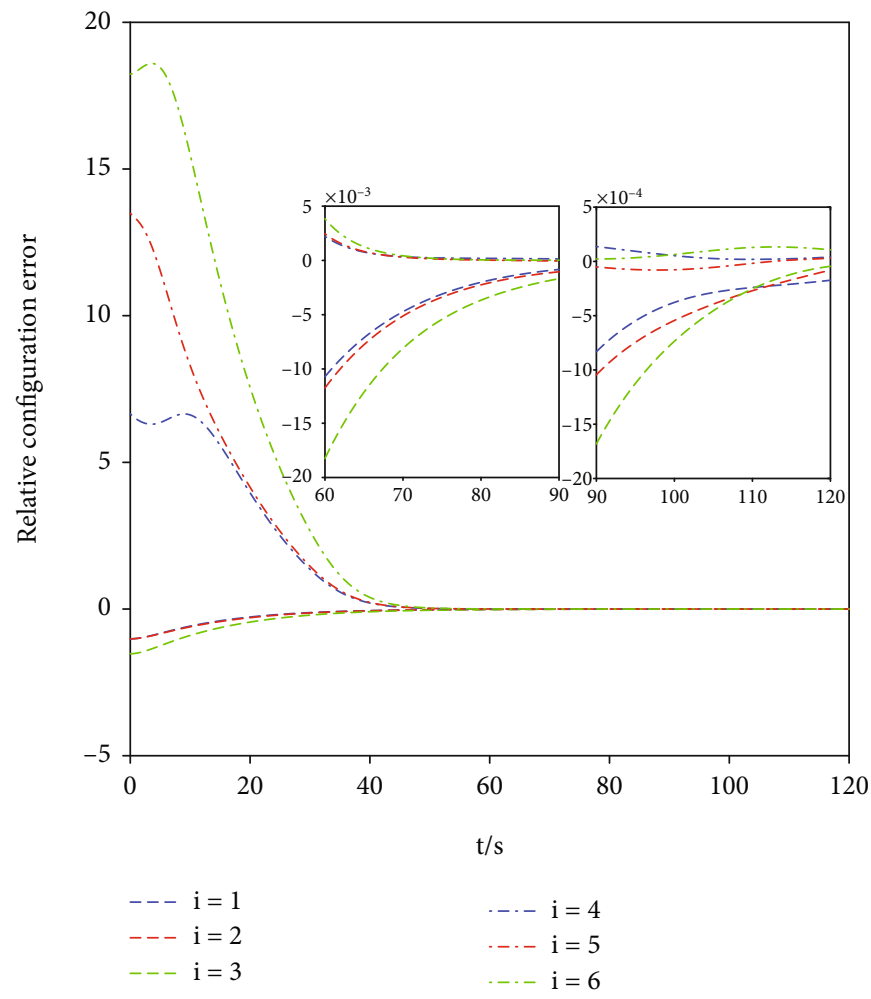

Figure 4: Relative configuration error curves.

the quantizer parameter (Equation (39a)). By applying these steps, the quantized finite-time controller is fulfilled.

The design process of the quantized finite-time TSMC is shown in Figure 3. More detailed analysis about the proposed method can be seen in Remarks 15-17.

Remark 15. Inspired by [32], a modified switched TSMS is designed in Equation (35) with the aid of a nonlinear piecewise smooth function. As has been illustrated in [32], $\vartheta=$ $((\pi / 2)(1-\kappa))^{1 /(1-\kappa)}$ is essential to ensure $\varphi\left(\tilde{\eta}_{i}\right)$ to be continuous and differentiable at point $\left|\tilde{\eta}_{i}\right|=\vartheta$. The sliding mode variable $S$ implemented here has two merits. Firstly, the terminal sliding mode variable can improve the convergence rate and guarantee the finite-time stability of the global system with improved robustness. In addition, the term $\varphi\left(\tilde{\eta}_{i}\right)$ defined by Equation (36) can avoid the singularity problem. Compared with the existing SMC methods in $[6,27,28]$, the proposed $S$ possesses the advantage of finite-time convergence with nonsingularity.

Remark 16. The control input in Equation (39) is designed to guarantee the system with finite-time convergence and convincing robustness. The designed control input $\varepsilon_{u}$ is derived by $u$ and $\delta$. More specifically, $k_{3} S$ is designed to guarantee the global stability of the system and $S_{\alpha}$ in Equation (39) is a finite-time convergent term which is different from the hyperbolic tangent function-based control input in $[5,6]$ and the Sigmoid function-based control input in [31, 32]. $\Gamma$ is composed of state parameters and adaptive parameters,

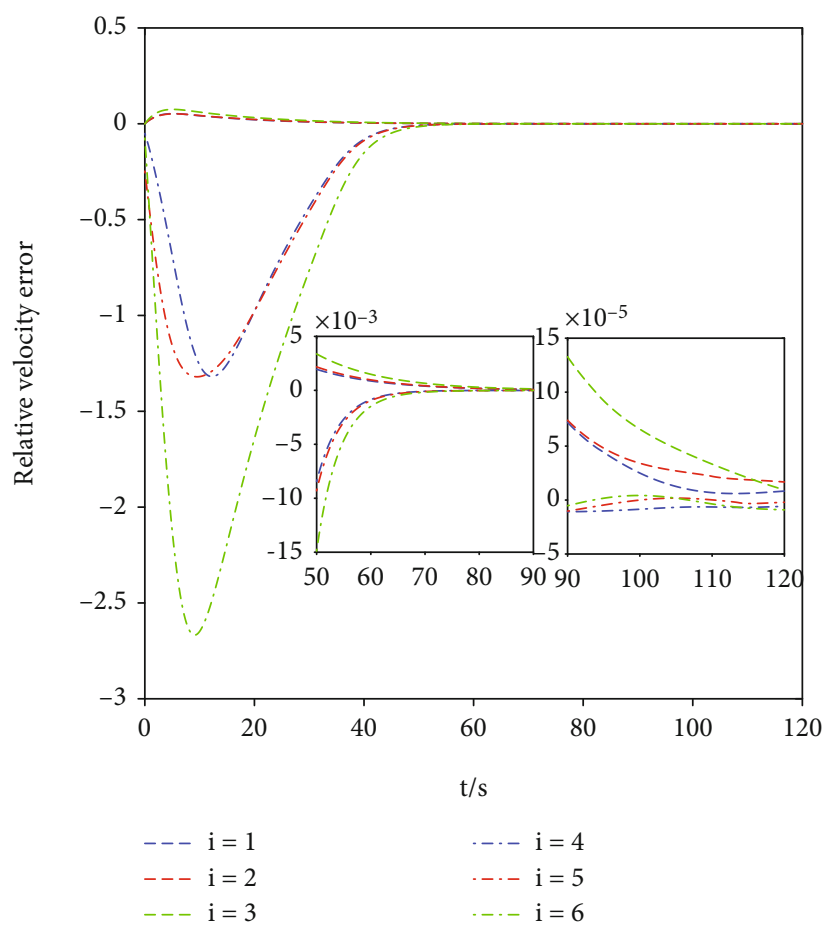

FIgURE 5: Relative velocity error curves.

which enjoys the property of improving system robustness and ensuring the convergence of adaptive parameters. It is worth noting that $u^{2} / \sqrt{D \wedge^{2}\|S\|^{2}+\varepsilon_{1}^{2}}$ in $\Gamma$ can reduce the quantization errors. The introduction of $S_{\alpha}$ and $\Gamma$ can 


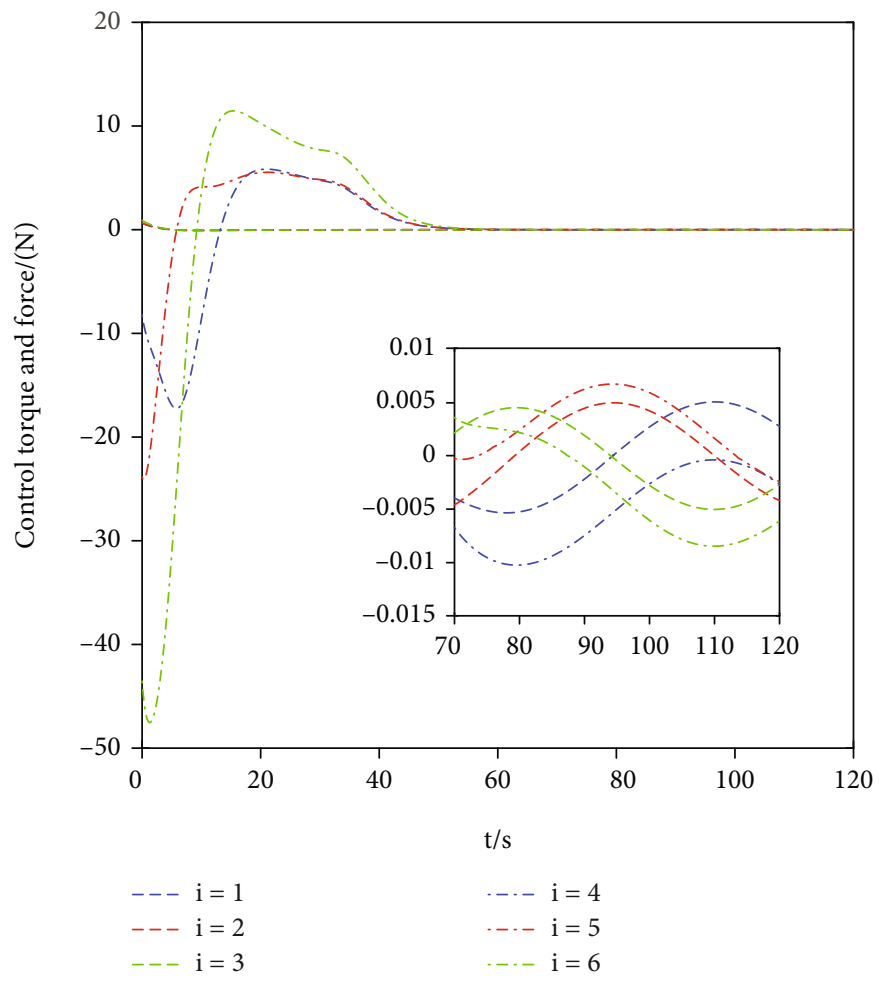

FIgURE 6: Control torque and force curves.

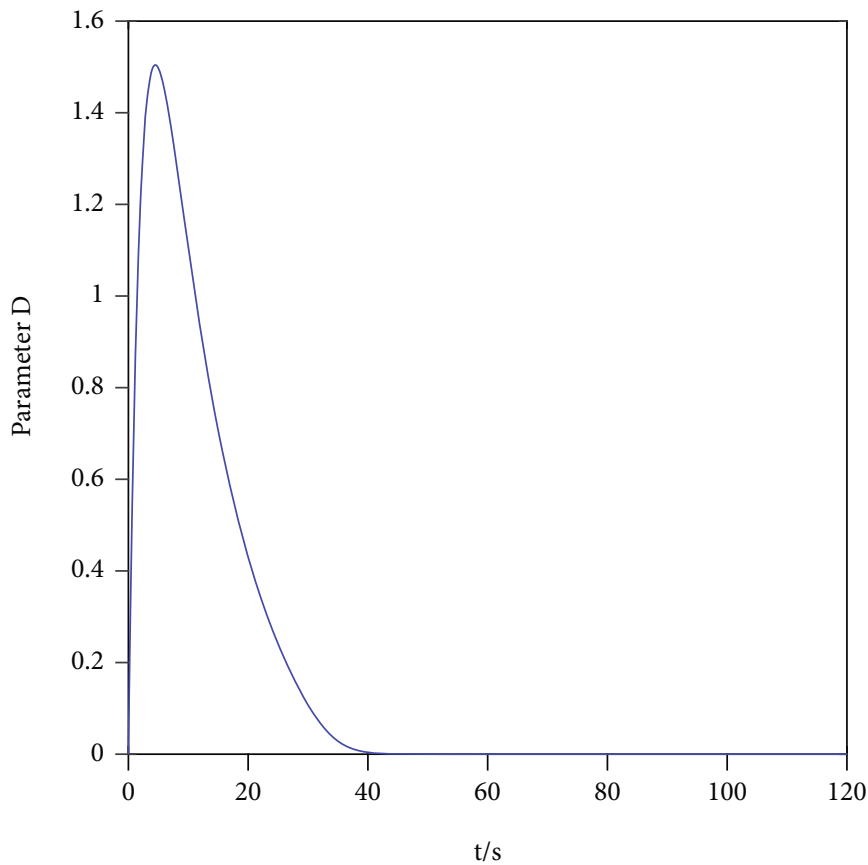

FIGURE 7: Estimated parameter curve.

improve the performance of system stability and avoid inherent chattering phenomenon.

Remark 17. There are seven control parameters in the finitetime controller, e.g., $k_{i}(i=1,2,3,4), c_{i}(i=1,2)$, and $\varepsilon_{1}$, which have an effect on the control performance of the final proximity maneuver system. Although the criterion to select appropriate parameters mainly consists in the rule of thumb, there are rules to follow. More specifically, a bigger value of $k_{i}(i=1,2,3,4)$ means higher control accuracy and bigger 


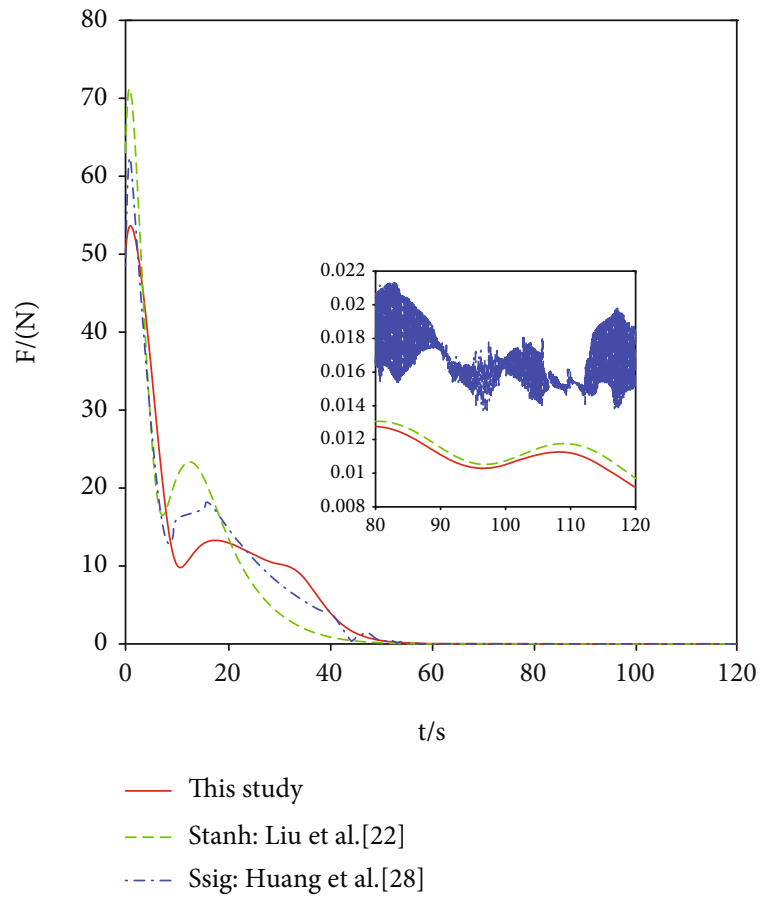

FIGURE 8: Control force curves.

overshoot of control force. The convergence rate of $(\tilde{\omega}, \tilde{\Theta})$ will increase as $k_{1}$ and $k_{2}$ increase while the settling time of $(\tilde{r}, \tilde{v})$ mainly depends on the value of $k_{3}$ and $k_{4}$. However, increasing $k_{2}$ will improve the settling time of $(\tilde{r}, \tilde{v})$ and increasing $k_{4}$ will aggravate the chattering phenomenon. Additionally, estimation errors mainly depend on $c_{i}(i=1,2)$ and a smaller $\varepsilon_{1}$ means higher accuracy. Given the above empirical rules, the approximate optimal solution can be found by trying various combination. In brief, the performance of the control system is determined by the controller, but also is largely affected by the design parameters.

Let $k_{i \text { min }}$ be the minimum of each dimension of $k_{i}$, that is, $k_{j \min }=\min \left(k_{j i}\right)$ with $i=1,2,3,4,5,6$ and $j=1,2,3,4$.

\subsection{Stability Analysis}

Theorem 18. Consider the relative accelerations of two spacecraft in RVD under Assumptions 11 and 12. The error dynamics model is modeled by Equation (23). The control schemes are designed as Equations (35)-(41) under $k_{i j} \geq 0$ $(i=1, \cdots, 4, j=1, \cdots, 6)$ and $c_{1}>0, c_{2}>0$. Under the proposed controller, the following conclusions can be obtained:

(i) The global system is finite-time stable, and all signals in the closed-loop system are guaranteed to be bounded. In particular, the TSMS $S$ will convergence to a small region $\Delta_{S}=\left\{\|S\| \mid\|S\|<\sqrt{2 / \lambda_{\Xi}}\left(\Delta_{1} / \rho\right)^{1 /(1+\alpha)}\right\}$ in finite time

(ii) The lumped errors $\tilde{\eta}_{i}$ and $\tilde{\xi}_{i}$ are convergent and will converge to the small region $\Delta_{\eta i}$ and $\Delta_{\xi i}$ in finite time, where $\Delta_{\eta}=\max \left\{\sqrt{2}\left(\Delta_{2} / \rho_{2}\right)^{1 /(\alpha+1)}, \vartheta\right\}$ and $\Delta_{\xi i}=$ $\max \left\{\vartheta_{1 i}, \vartheta_{2 i}\right\}$ with $\vartheta_{1 i}=\Delta_{S}+\left(k_{1 i}+\left(2 k_{2 i} / \pi\right)\right) \vartheta+k_{2 i}$ $\beta \vartheta^{\beta}$ and $\vartheta_{2 i}=\Delta_{S}+k_{1 i} \vartheta_{0}+k_{2 i} \vartheta_{0}^{\beta+1}$

Proof. Define the Lyapunov function (LF) candidate:

$$
V_{1}=\frac{1}{2} S^{\mathrm{T}} \Xi S
$$

Taking the time derivative of Equation (42) yields

$$
\begin{aligned}
\dot{V}_{1}= & S^{\mathrm{T}} \Xi \dot{S}=S^{\mathrm{T}} \Xi\left[\dot{\tilde{\xi}}+k_{1} \dot{\tilde{\eta}}+k_{2}(\dot{\varphi}(\tilde{\eta}))\right] \\
& =S^{\mathrm{T}}\left[\operatorname{ad}_{\xi}^{*} \Xi \xi+\varphi^{g}+Q_{\varphi}+\varphi^{d}+\Xi\left(\operatorname{ad}_{\xi} \operatorname{Ad}_{h^{-1}} \xi_{t}-\operatorname{Ad}_{h^{-1}} \dot{\xi}_{t}\right)\right] \\
& +S^{\mathrm{T}}\left[k_{1} \Xi G(\tilde{\eta}) \tilde{\xi}+k_{2}(\dot{\varphi}(\tilde{\eta}))\right] \\
& =S^{\mathrm{T}}\left[Q_{\varphi}+\varphi^{d}+\Lambda_{\varphi}+k_{1} \Xi G(\tilde{\eta}) \tilde{\xi}+k_{2}(\dot{\varphi}(\tilde{\eta}))\right] .
\end{aligned}
$$

Based on Equation (34), $S^{\mathrm{T}} Q_{\varphi}$ can be extended to be

$$
\begin{aligned}
S^{\mathrm{T}} Q_{\varphi}= & S^{\mathrm{T}}\left[\kappa(u) \boldsymbol{\varepsilon}_{u}+E(u) I_{1}\right] \\
& =S^{\mathrm{T}}\left[-\frac{\kappa(u)}{1-\delta} u+E(u) I_{1}\right] \leq-S^{\mathrm{T}} u+u_{\min } S^{\mathrm{T}} I_{1}
\end{aligned}
$$

Substituting Equation (44), the derivative of $V_{1}$ becomes

$$
\begin{aligned}
\dot{V}_{1} \leq & -S^{\mathrm{T}} u+u_{\min } S^{\mathrm{T}} I_{1}+S^{\mathrm{T}}\left[\varphi^{d}+\Lambda_{\varphi}+k_{1} \Xi G(\tilde{\eta}) \tilde{\xi}+k_{2}(\dot{\varphi}(\tilde{\eta}))\right] \\
\leq & -S^{\mathrm{T}}\left(k_{1} \Xi G(\tilde{\eta}) \tilde{\xi}+k_{2}(\dot{\varphi}(\tilde{\eta}))\right)-S^{\mathrm{T}} k_{3} S-\frac{S^{\mathrm{T}} k_{4} S_{\alpha}}{\sqrt{\|S\|^{2(\alpha+1)}+\varepsilon_{1}^{2}}}+u_{\min } S^{\mathrm{T}} I_{1} \\
+ & S^{\mathrm{T}}\left[\varphi^{d}+\Lambda_{\varphi}+k_{1} \Xi G(\tilde{\eta}) \tilde{\xi}+k_{2}(\dot{\varphi}(\tilde{\eta}))\right]-\frac{\left\|\Lambda_{\varphi}\right\|^{2} S^{\mathrm{T}} S}{\sqrt{\left\|\Lambda_{\varphi}\right\|^{2}\|S\|^{2}+\varepsilon_{1}^{2}}} \\
& -\frac{D \wedge^{2} S^{\mathrm{T}} S}{\sqrt{D \wedge^{2}\|S\|^{2}+\varepsilon_{1}^{2}}}-\frac{u^{2} S^{\mathrm{T}} S}{\sqrt{D \wedge^{2}\|S\|^{2}+\varepsilon_{1}^{2}}} \\
\leq & -k_{3 \min }\|S\|^{2}-k_{4 \min }\|S\|^{(\alpha+1)}-\|S\|\left\|\Lambda_{\varphi}\right\|-\hat{D}\|S\|-u\|S\|+\mu_{\min }\|S\|+D\|S\| \\
& +S^{\mathrm{T}} \Lambda_{\varphi}+k_{4 \min } \varepsilon_{1}+\varepsilon_{1}+\varepsilon_{1}+\varepsilon_{1} \\
\leq & -k_{3 \min }\|S\|^{2}-k_{4 \min }\|S\|^{(\alpha+1)}+\tilde{D}\|S\|+\left(3+k_{4 \min }\right) \varepsilon_{1} .
\end{aligned}
$$

Define the overall LF candidate:

$$
V_{2}=V_{1}+\frac{1}{2 c_{1}} \tilde{D}^{2}
$$

Taking the time derivative of Equation (46) yields

$$
\begin{aligned}
\dot{V}_{2} \leq & -k_{3 \min }\|S\|^{2}-k_{4 \min }\|S\|^{(\alpha+1)}+\tilde{D}\|S\|+\frac{1}{c_{1}} \tilde{D} \dot{\tilde{D}} \\
& +\left(3+k_{4 \min }\right) \varepsilon_{1}=-k_{3 \min }\|S\|^{2}-k_{4 \min }\|S\|^{(\alpha+1)}+\tilde{D}\|S\| \\
& -\frac{1}{c_{1}} \tilde{D} \dot{\hat{D}}+\left(3+k_{4 \min }\right) \varepsilon_{1}=-k_{3 \min }\|S\|^{2}-k_{4 \min }\|S\|^{(\alpha+1)}
\end{aligned}
$$




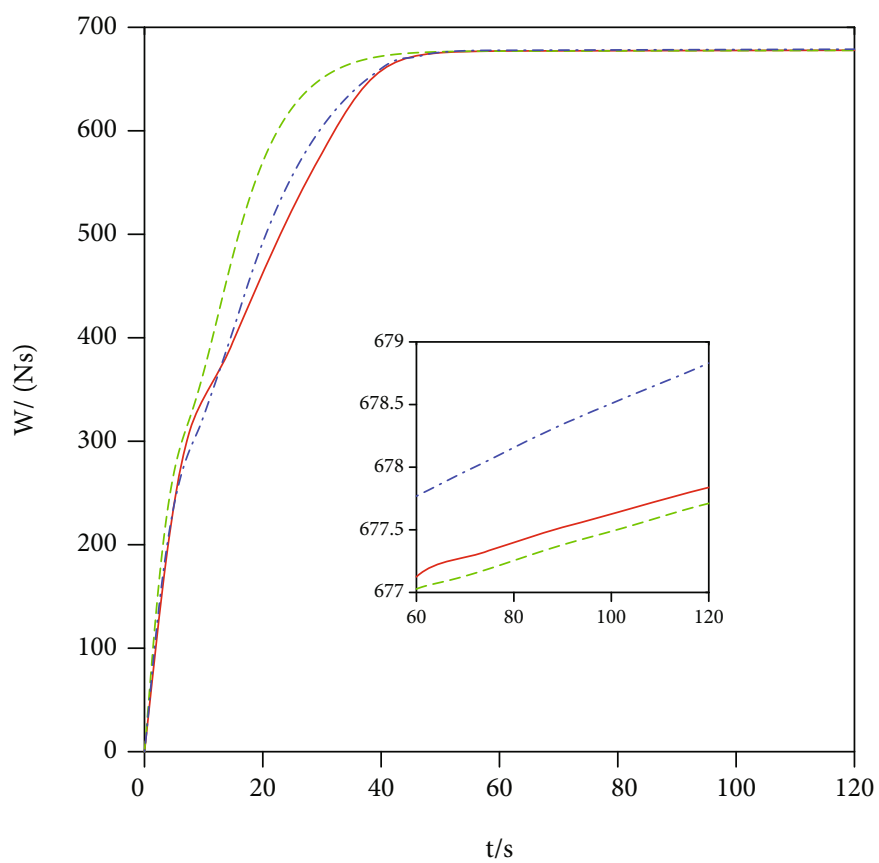

— This study

- - - Stanh: Liu et al.[22]

-..- Ssig: Huang et al.[28]

FIgURE 9: Curves of the integration of control force.
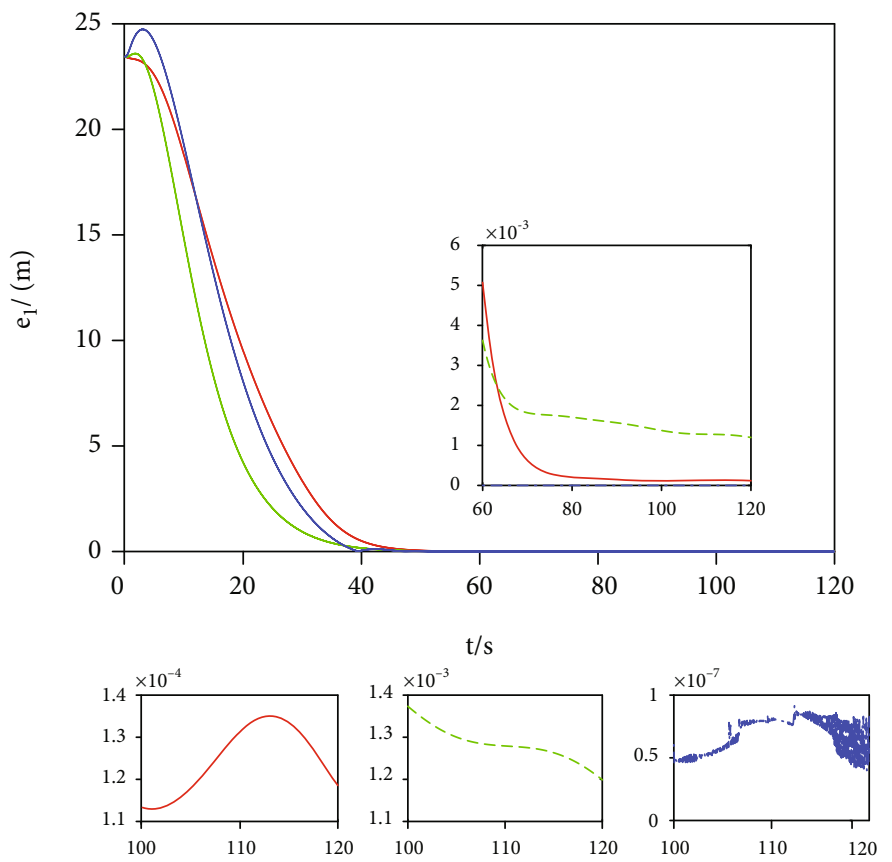

— This study

- - Stanh: Liu et al.[22]

-..- Ssig: Huang et al.[28]

Figure 10: Relative distance error curves. 


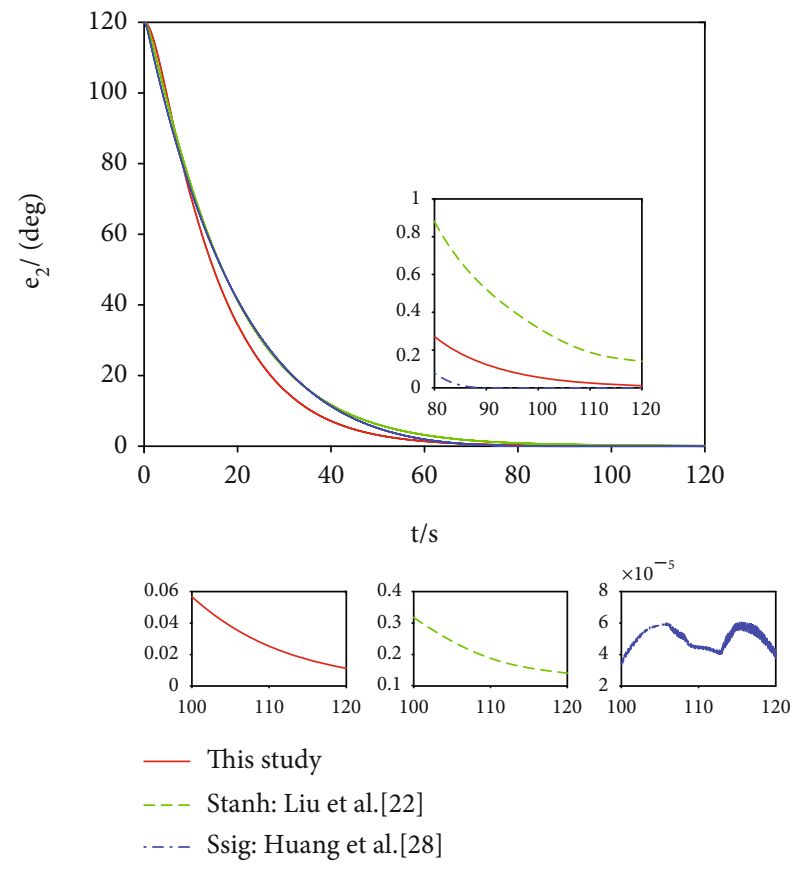

FIgURE 11: Relative attitude error curves.

In consideration of Lemma 8 and $\alpha$ defined in Equation (30), the following equation holds:

$$
\begin{aligned}
\tilde{D} \widehat{D}= & \tilde{D} \\
& =-\frac{1}{4}|\tilde{D}|^{2}-\frac{1}{4}\left(|\tilde{D}|-|\tilde{D}|^{\alpha}\right)^{2}+\frac{1}{4}|\tilde{D}|^{2 \alpha}-\frac{1}{2}|\tilde{D}|^{(1+\alpha)} \\
& +\frac{1}{2} D^{2} \leq-\frac{1}{2}|\tilde{D}|^{(1+\alpha)}+\frac{1}{2} D^{2}-\frac{1}{4}|\tilde{D}|^{2}+\frac{1}{4}|\tilde{D}|^{2 \alpha} \\
& \leq-\frac{1}{2}|\tilde{D}|^{(1+\alpha)}+\frac{1}{2} D^{2}+\frac{1-\alpha}{4 \alpha^{(\alpha /(1-\alpha))}} .
\end{aligned}
$$

Substituting Equation (48) into Equation (47) yields

$$
\begin{aligned}
\dot{V}_{2} \leq & -k_{3 \min }\|S\|^{2}-k_{4 \min }\|S\|^{(\alpha+1)}+\left(3+k_{4 \min }\right) \varepsilon_{1} \\
& +c_{2}\left(-\frac{1}{2}|\tilde{D}|^{(1+\alpha)}+\frac{1}{2} D^{2}+\frac{1-\alpha}{4 \alpha^{(\alpha /(1-\alpha))}}\right) \\
& =-k_{3 \min }\|S\|^{2}-k_{4 \min }\|S\|^{(\alpha+1)}-\frac{c_{2}}{2}|\tilde{D}|^{(1+\alpha)}+\Delta_{1},
\end{aligned}
$$

where $\Delta_{1}=\left(c_{2} / 2\right)\left(2 D^{2}+\left((1-\alpha) / \alpha^{(\alpha /(1-\alpha))}\right)\right)+\left(3+k_{4 \min }\right) \varepsilon_{1}$.

Through mathematical transformation, Equation (49) can be rewritten as

$$
\begin{aligned}
\dot{V}_{2} \leq & -k_{4 \min }\left(S^{\mathrm{T}} S\right)^{(1+\alpha) / 2}-\frac{c_{2}}{2}\left(|\tilde{D}|^{2}\right)^{(1+\alpha) / 2}+\Delta_{1} \\
& =-k_{4 \min }\left(S^{\mathrm{T}} S\right)^{(1+\alpha) / 2}-\frac{c_{2}}{2}\left(\tilde{D}^{2}\right)^{(1+\alpha) / 2}+\Delta_{1} \\
& =-k_{4 \min }\left(\frac{2}{\lambda_{\max }(\Xi)}\right)^{(1+\alpha) / 2}\left(\frac{1}{2} S^{\mathrm{T}} \Xi S\right)^{(1+\alpha) / 2} \\
& -\frac{c_{2}}{2}\left(2 c_{1}\right)^{(1+\alpha) / 2}\left(\frac{1}{2 c_{1}} \tilde{D}^{2}\right)^{(1+\alpha) / 2} \\
& +\Delta_{1} \leq-\rho V_{2}^{((1+\alpha) / 2)}+\Delta_{1}
\end{aligned}
$$

where $\rho=\min \left\{k_{4 \min }\left(2 / \lambda_{\Xi}\right)^{(1+\alpha) / 2},\left(c_{2} / 2\right)\left(2 c_{1}\right)^{(1+\alpha) / 2}\right\}$ with $\lambda_{\Xi}$ being the minimum of each dimension of $\Xi$. It is obvious that $\rho>0, \Delta_{1} \geq 0$.

Remark 19. As mentioned in Remark 10, it is known that $\alpha=-m /(2 n+1)$ with $0<2 m<2 n+1$. As a result, $\tilde{D}^{\alpha}$ and $\tilde{D}^{(1+2 \alpha)}$ will make sense. It concludes that $|\tilde{D}|^{(1+\alpha)}=$ $\left(|\tilde{D}|^{2}\right)^{(1+\alpha) / 2}=\left(\tilde{D}^{2}\right)^{(1+\alpha) / 2}$. Furthermore, $1 / 4<(1+\alpha) / 2<1 / 2$ is tenable such that $\dot{V}_{2} \leq-\rho V_{2}^{((1+\alpha) / 2)}+\Delta_{1}$ in Equation (50) satisfies the requirement in Lemma 9. As a consequence, the system is finite-time stable.

Equation (50) can be reconstructed to be

$$
\dot{V}_{2} \leq-\rho V_{2}^{((1+\alpha) / 2)}+\Delta_{1}=-\left(\rho-\frac{\Delta_{1}}{V_{2}^{((1+\alpha) / 2)}}\right) V_{2}^{((1+\alpha) / 2)}
$$

As the result in Equation (50) has proved that the global system is stable, thus, $V_{2}^{((1+\alpha) / 2)} \geq \Delta_{1} / \rho$ holds until the system turning to be stable. Consequently, it yields the convergence region of $S: \Delta_{S}=\left\{\|S\| \mid\|S\|<\sqrt{2 / \lambda_{\Xi}}\left(\Delta_{1} / \rho\right)^{1 /(1+\alpha)}\right\}$..

Thus, point (i) has been proven.

Remark 20. Through the design of the controller and the proof of the effectiveness, the result in Equation (50) indicates that the system is globally stable with finite-time convergence. Significantly, $V_{2}$ is only the LF candidate formed by $S$ and $\tilde{D}$. The stability of system states $(\tilde{\xi}$ and $\tilde{\eta})$ is not clear. Thus, a further developed analysis and proof are needed.

Since $S=\left[\begin{array}{llllll}S_{1} & S_{2} & S_{3} & S_{4} & S_{5} & S_{6}\end{array}\right]^{\mathrm{T}}$ satisfies $\|S\| \leq \Delta_{S}$, one has $S_{i}=\tilde{\xi}_{i}+k_{1 i} \tilde{\eta}_{i}+k_{2 i} \varphi\left(\tilde{\eta}_{i}\right)=\vartheta_{S}$ with $\vartheta_{S} \in\left[-\Delta_{S}, \Delta_{S}\right]$.

When $\left|\tilde{\eta}_{i}\right| \leq \vartheta, \quad \tilde{\xi}_{i}+k_{1 i} \tilde{\eta}_{i}+k_{2 i}\left[(2 \vartheta / \pi) \sin \left((\pi / 2 \vartheta) \tilde{\eta}_{i}\right)+\right.$ $\left.\beta \vartheta^{\beta-1} \tilde{\eta}_{i}\right] \leq \Delta_{S}$ holds all time, which implies

$$
\begin{aligned}
\left|\tilde{\xi}_{i}\right| \leq & \Delta_{S}+k_{1 i}\left|\tilde{\eta}_{i}\right|+k_{2 i}\left[\frac{2 \vartheta}{\pi} \sin \left(\frac{\pi}{2 \vartheta}\left|\tilde{\eta}_{i}\right|\right)+\beta \vartheta^{\beta-1}\left|\tilde{\eta}_{i}\right|\right] \\
& \leq \Delta_{S}+k_{1 i} \vartheta+k_{2 i}\left[\frac{2 \vartheta}{\pi} \sin \left(\frac{\pi}{2 \vartheta} \vartheta\right)+\beta \vartheta^{\beta-1} \vartheta\right] \\
& =\Delta_{S}+\left(k_{1 i}+\frac{2 k_{2 i}}{\pi}\right) \vartheta+k_{2 i} \beta \vartheta^{\beta}=\vartheta_{1 i} .
\end{aligned}
$$

When $\left|\tilde{\eta}_{i}\right|>\vartheta$, the LF is chosen as

$$
V_{0}=\sum_{i=1}^{6} \frac{1}{2} \tilde{\eta}_{i}^{2}
$$



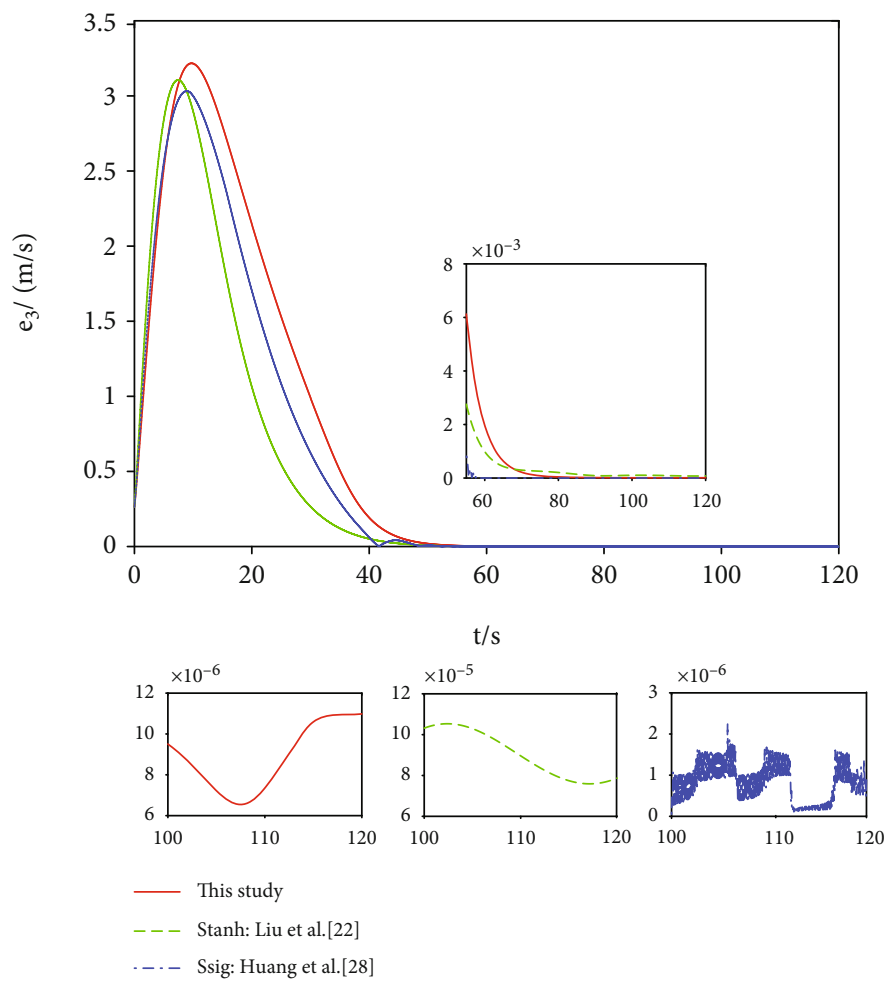

-..- Ssig: Huang et al.[28]

FIGURE 12: Relative translational velocity error curves.
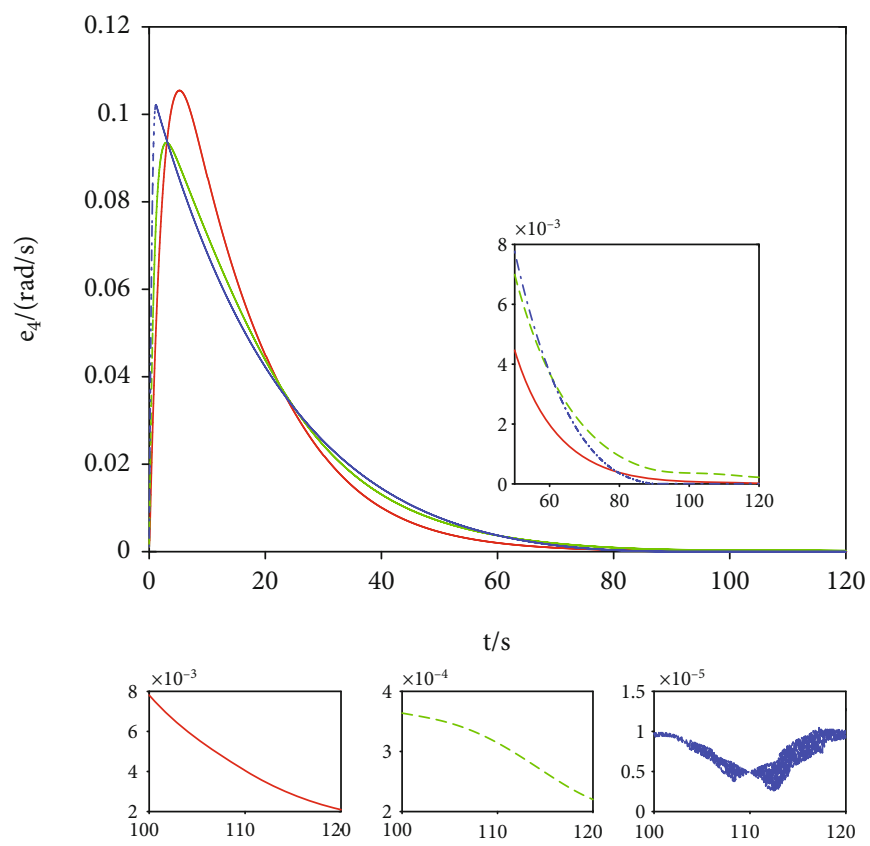

— This study

- - - Stanh: Liu et al.[22]

-... Ssig: Huang et al.[28]

FIGURE 13: Relative angular velocity error curves. 


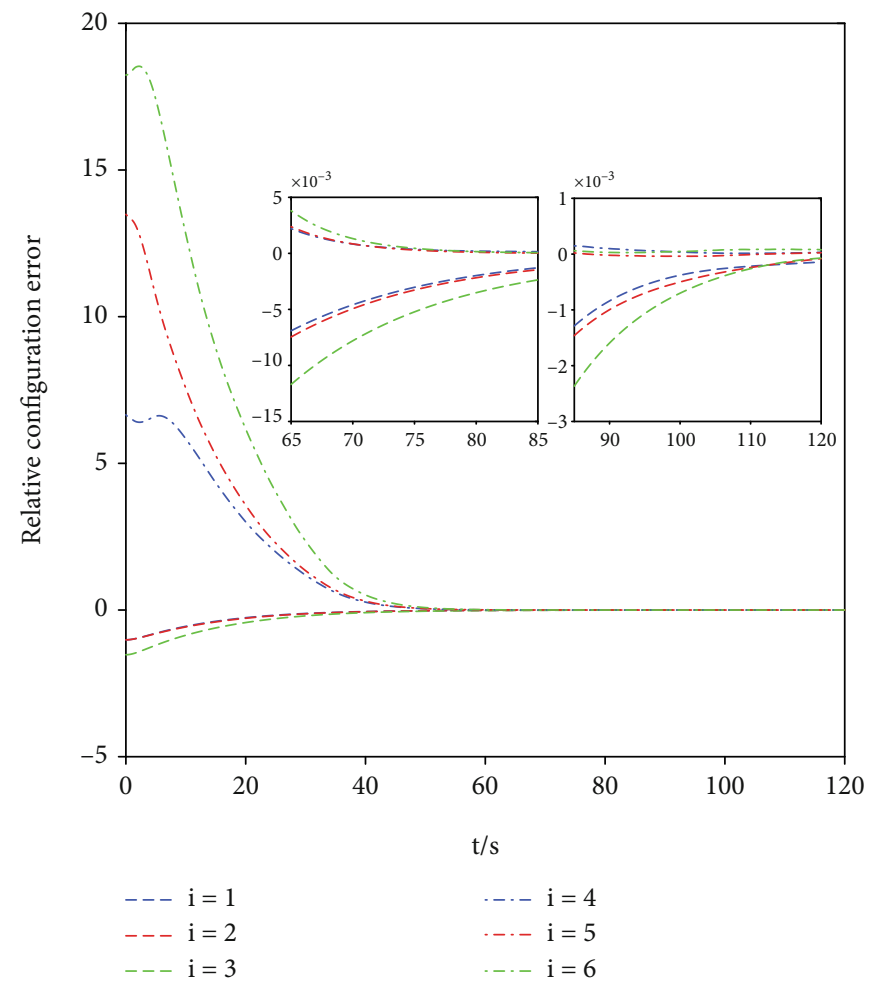

FIGURE 14: Relative configuration error curves.

Taking the time derivate of $V_{0}$ yields

$$
\begin{aligned}
\dot{V}_{0}= & \sum_{i=1}^{6} \tilde{\eta}_{i} G\left(\tilde{\eta}_{i}\right) \tilde{\xi}_{i}=\sum_{i=1}^{6} \tilde{\eta}_{i}\left(\vartheta_{S}-k_{1 i} \tilde{\eta}_{i}-k_{2 i} \varphi\left(\tilde{\eta}_{i}\right)\right) \\
& =\sum_{i=1}^{6}\left(\vartheta_{S} \tilde{\eta}_{i}-k_{1 i} \tilde{\eta}_{i}^{2}-k_{2 i}\left|\tilde{\eta}_{i}\right|^{\beta+1}\right) \\
& \leq \sum_{i=1}^{6}\left(-k_{1 i} \tilde{\eta}_{i}^{2}-k_{2 i}\left|\tilde{\eta}_{i}\right|^{\beta+1}+k_{1 i} \tilde{\eta}_{i}^{2}+\frac{1}{4 k_{1 i}} \vartheta_{S}^{2}\right) \\
& =\sum_{i=1}^{6}\left(-k_{2 i}\left|\tilde{\eta}_{i}\right|^{\beta+1}+\frac{1}{4 k_{1 i}} \vartheta_{S}^{2}\right) \leq-\rho_{1} V_{0}^{(\beta+1) / 2}+\Delta_{2},
\end{aligned}
$$

with $\Delta_{2}=\sum_{i=1}^{6}\left(\vartheta_{S}^{2} / 4 k_{1 i}\right)$ and $\rho_{1}=k_{2} \min 2^{(\beta+1) / 2}$, which implies that the LF $V_{0}$ is stable when $\left|\tilde{\eta}_{i}\right|>\vartheta$. Through the result in Equation (54), one has

$$
\dot{V}_{0} \leq-\left(\rho_{1}-\frac{\Delta_{2}}{V_{0}^{(\beta+1) / 2}}\right) V_{0}^{(\beta+1) / 2}
$$

Based on Equation (55), if $\rho_{1}-\left(\Delta_{2} / V_{0}^{(\beta+1) / 2}\right) \geq 0$ holds, $\tilde{\eta}_{i}$ will converge to the region $\left|\tilde{\eta}_{i}\right|<\sqrt{2}\left(\Delta_{2} / \rho_{1}\right)^{1 /(\beta+1)}$ in finite time. Consequently, $\tilde{\eta}_{i}$ will converge to the region $\Delta_{\eta}$.
Let $\vartheta_{0}=\sqrt{2}\left(\Delta_{2} / \rho_{2}\right)^{1 /(\beta+1)}$. According to Equation (51), $\tilde{\xi}_{i}+k_{1 i} \tilde{\eta}_{i}+k_{2 i}\left|\tilde{\eta}_{i}\right|^{\beta+1} \leq \Delta_{S}$ holds, which implies

$$
\begin{gathered}
\left|\tilde{\xi}_{i}\right| \leq \Delta_{S}+k_{1 i}\left|\tilde{\eta}_{i}\right|+k_{2 i}\left|\tilde{\eta}_{i}\right|^{\beta+1} \\
\leq \Delta_{S}+k_{1 i} \vartheta_{0}+k_{2 i} \vartheta_{0}^{\beta+1}=\vartheta_{2 i} .
\end{gathered}
$$

Consequently, $\tilde{\xi}_{i}$ will converge to the region $\Delta_{\xi i}=\max$ $\left\{\vartheta_{1 i}, \vartheta_{2 i}\right\}$ with $\vartheta_{1 i}=\Delta_{S}+\left(k_{1 i}+\left(2 k_{2 i} / \pi\right)\right) \vartheta+k_{2 i} \beta \vartheta^{\beta}$ and $\vartheta_{2 i}=$ $\Delta_{S}+k_{1 i} \vartheta_{0}+k_{2 i} \vartheta_{0}^{\beta+1}$.

Thus, point (ii) has been proven.

Finally, Theorem 18 has been proven.

\section{Simulation Results}

In this section, numerical simulations are developed to validate the effectiveness of the proposed scheme for spacecraft RVD maneuver control. A RVD scenario with a chaser and a target is adopted in this simulation. The target is assumed to maneuver freely in an elliptical orbit (the target will not maneuver or be affected by space interference). The chaser is forced to track the target to achieve the objective. According to [1], the inertia parameters of the target and the chaser are given as 
TABLE 6: Control precision comparisons.

\begin{tabular}{|c|c|c|c|c|}
\hline Component & & $S_{\text {proposed }}$ & $S_{\text {tanh }}$ & $S_{\text {sig }}$ \\
\hline \multirow{2}{*}{ Relative distance error } & Accuracy (m) & $5 \times 10^{-3}$ & $9 \times 10^{-3}$ & $9 \times 10^{-3}$ \\
\hline & Time (s) & 60 & 55 & 50 \\
\hline \multirow{2}{*}{ Relative attitude error } & Accuracy (deg) & 0.6 & 0.9 & 0.6 \\
\hline & Time $(s)$ & 70 & 80 & 70 \\
\hline \multirow{2}{*}{ Relative translational velocity error } & Accuracy $(\mathrm{m} / \mathrm{s})$ & $6 \times 10^{-3}$ & $8 \times 10^{-3}$ & $7 \times 10^{-3}$ \\
\hline & Time (s) & 55 & 50 & 50 \\
\hline \multirow{2}{*}{ Relative angular velocity error } & Accuracy (deg/s) & $7 \times 10^{-3}$ & $7 \times 10^{-3}$ & $8 \times 10^{-3}$ \\
\hline & Time (s) & 45 & 50 & 50 \\
\hline
\end{tabular}

$$
\begin{aligned}
& \text { Chaser : } m=100 \mathrm{~kg}, J=\left[\begin{array}{rrr}
23.7 & -0.2 & -0.5 \\
-0.2 & 25.3 & -0.3 \\
-0.5 & -0.3 & 22.5
\end{array}\right] \mathrm{kg} \cdot \mathrm{m}^{2}, \\
& \text { Target : } m_{t}=110 \mathrm{~kg}, J_{t}=\left[\begin{array}{rrr}
21.7 & -0.2 & -0.5 \\
-0.2 & 22.3 & -0.3 \\
-0.5 & -0.3 & 25.3
\end{array}\right] \mathrm{kg} \cdot \mathrm{m}^{2} \text {. }
\end{aligned}
$$

$$
R_{t 0}=\left[\begin{array}{ccc}
\cos (\Omega) \cos (\omega)-\sin (\Omega) \cos (i) \sin (\omega) & -\cos (\Omega) \sin (\omega)-\sin (\Omega) \cos (i) \cos (\omega) & \sin (\Omega) \sin (i) \\
\sin (\Omega) \cos (\omega)+\cos (\Omega) \cos (i) \sin (\omega) & -\sin (\Omega) \sin (\omega)+\cos (\Omega) \cos (i) \cos (\omega) & -\cos (\Omega) \sin (i) \\
\sin (i) \sin (\omega) & \sin (i) \cos (\omega) & \cos (i)
\end{array}\right]
$$

The initial orbital elements of the target are given in Table $1[1,43]$. As the chaser is forced to rendezvous with the target, whose initial states relative to the target are essential and given in Table $2[1,43]$. It assumes that $\left\{B_{t}\right\}$ is in the same direction as LVLH of the target at the beginning of the mission. Consequently, the initial transfer matrix $R_{t 0}$ is as [1]:

The time varying disturbance $\varphi^{d}=\left[\begin{array}{ll}\tau_{d} & \Phi_{d}\end{array}\right]^{\mathrm{T}}$ is defined

$$
\begin{array}{rlr}
\tau_{d} & =0.005 \times\left[\begin{array}{lll}
\sin (0.1 t) & \cos (0.1 t) & -\sin (0.1 t)
\end{array}\right]^{\mathrm{T}}, \\
\Phi_{d} & =0.005 \times\left[\begin{array}{lll}
\sin (0.1 t) & \cos (0.1 t) & -\sin (0.1 t)
\end{array}\right]^{\mathrm{T}} .
\end{array}
$$

The purpose of error tracking control is to drive the relative states to track the desired one. The desired states are given in Table $3[1,43]$.

The simulation result includes two parts: (1) the comparison of three different kinds of SMC algorithms and (2) the verification of the proposed quantized TSMC scheme.

4.1. Simulation Results of Three Finite-Time Control Schemes without Input Quantization. To better show the superiority of the proposed control algorithm, three different controllers are simulated. Input quantization is not considered in this section.

Inspired by the proposed controller in Equation (39), the newly designed control input is $u=-k_{1} \Xi G(\tilde{\eta}) \tilde{\xi}-k_{2}(\dot{\varphi}(\tilde{\eta}))$ 


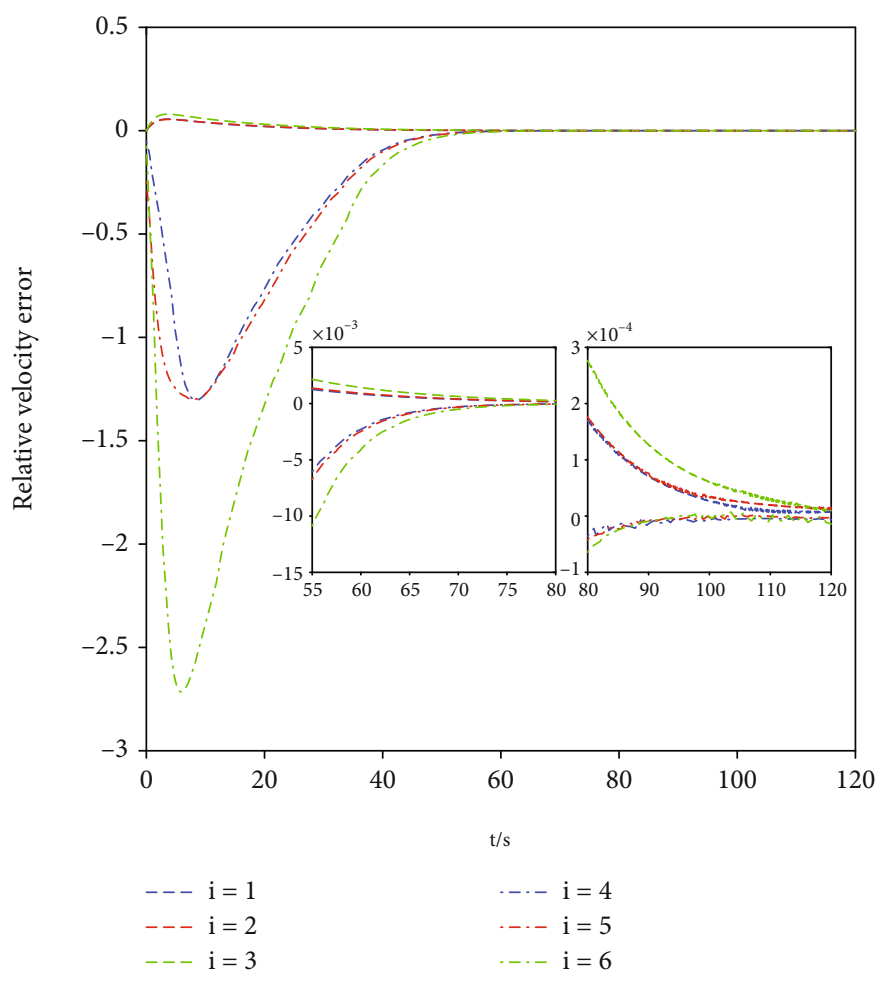

FIGURE 15: Relative velocity error curves.

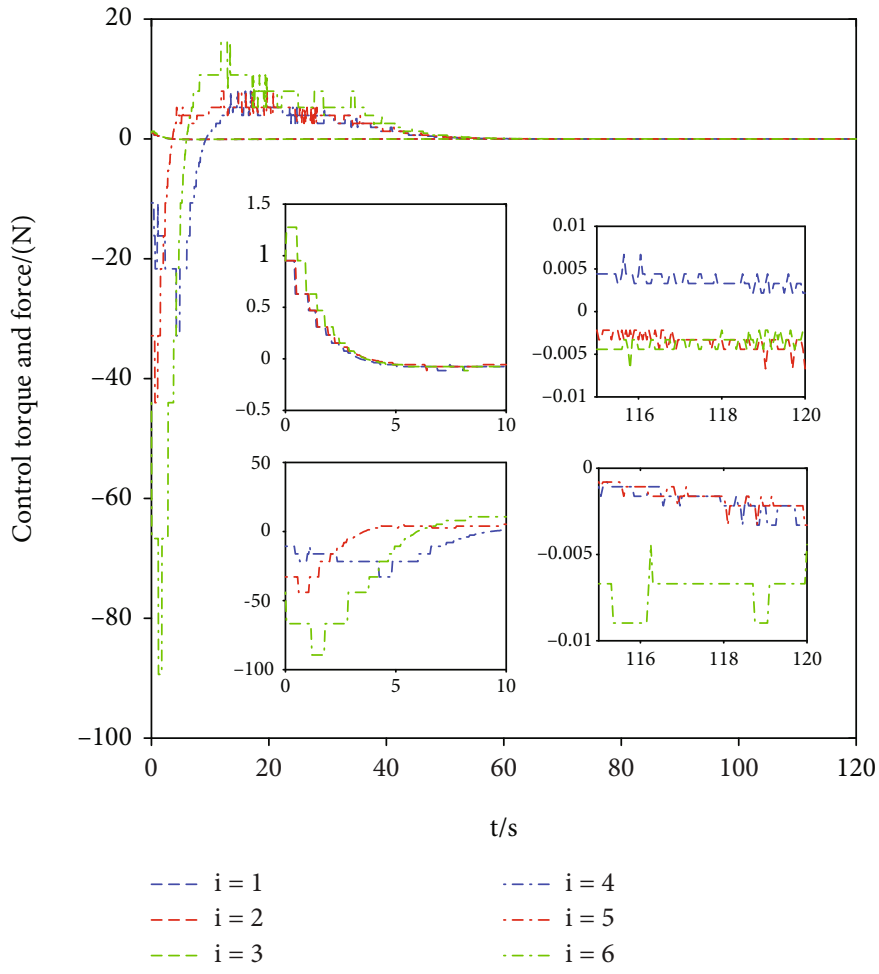

FIgURE 16: Control torque and force curves. 


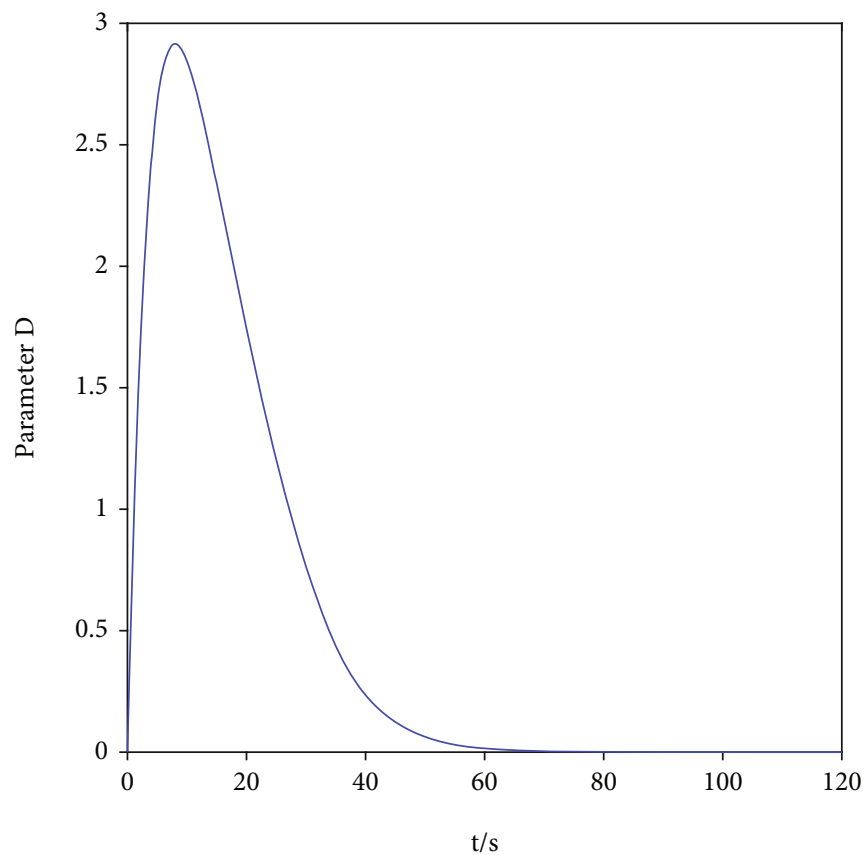

Figure 17: Estimated parameter.

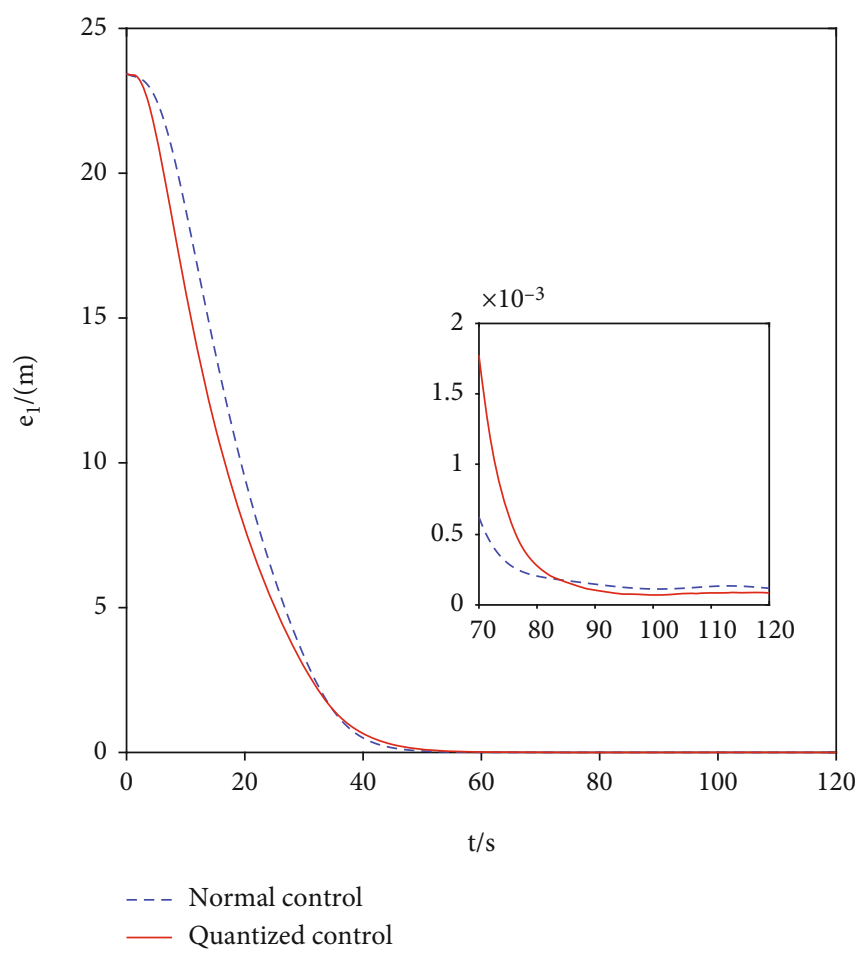

FIgURE 18: Relative distance error curves.

The simulation results of $S_{\text {proposed }}$ are presented in Figures $4-7$. And the contrastive results between $S_{\text {proposed }}$, $S_{\text {sig }}$, and $S_{\text {tanh }}$ are shown in Figures 8-13.

Figures 4 and 5 indicate the relative configuration error and relative velocity error under the proposed control scheme, respectively. In Figure 4 , the curves of $i=1,2,3$ denote the attitude tracking error and curves of $i=4,5,6$ denote the position tracking error. According to Figure 4, the attitude tracking error converges to less than $10^{-3} \times$ $\left[\begin{array}{lll}5 & 6 & 7\end{array}\right]^{\mathrm{T}} \mathrm{rad}$ in $70 \mathrm{~s}$ and the position tracking error 


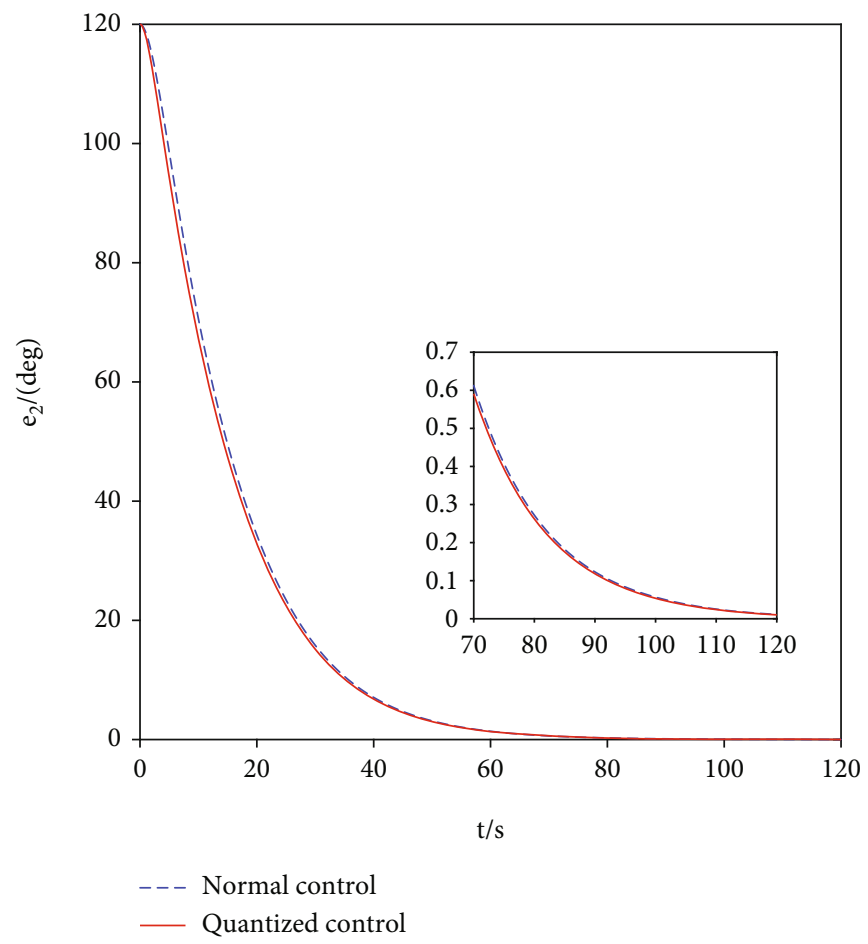

Figure 19: Relative attitude error curves.

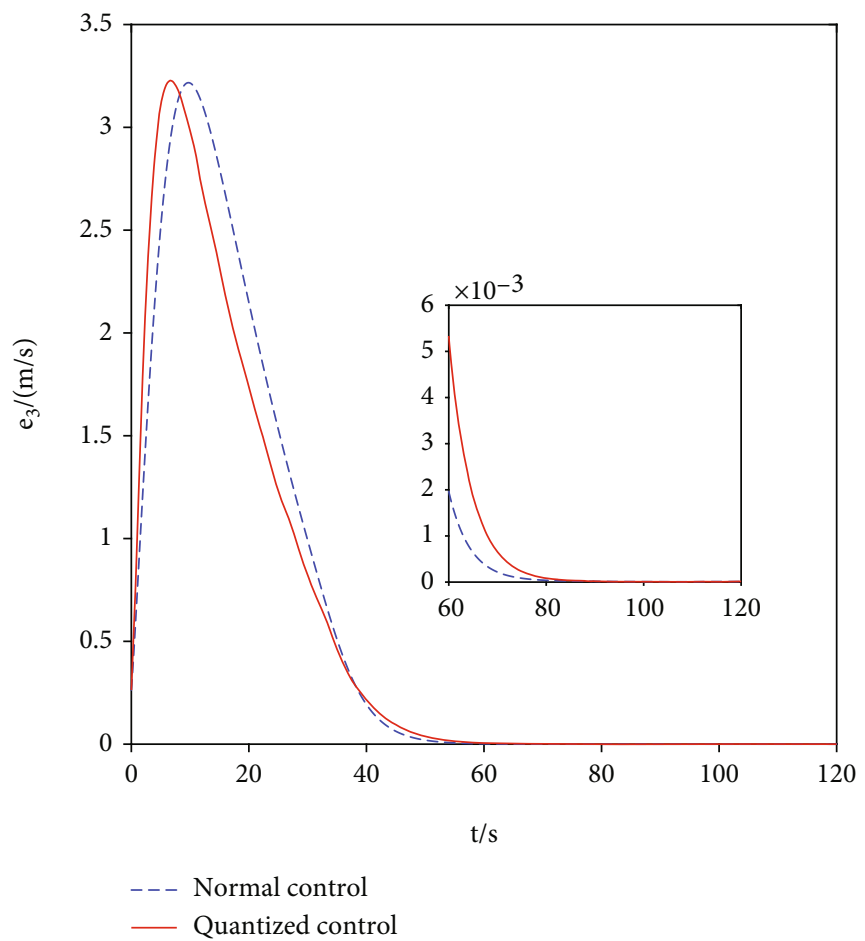

FIGURE 20: Relative translational velocity error curves.

converges to less than $10^{-3} \times\left[\begin{array}{lll}3 & 3 & 4\end{array}\right]^{\mathrm{T}} \mathrm{m}$ in $60 \mathrm{~s}$. In Figure 5, the curves of $i=1,2,3$ denote the angular velocity tracking error and curves of $i=4,5,6$ denote the transla- tional velocity tracking error. According to Figure 5, the angular velocity tracking error converges to less than $10^{-3}$ $\times\left[\begin{array}{lll}5 & 5 & 8\end{array}\right]^{\mathrm{T}} \mathrm{rad} / \mathrm{s}$ in $40 \mathrm{~s}$ and the translational velocity 


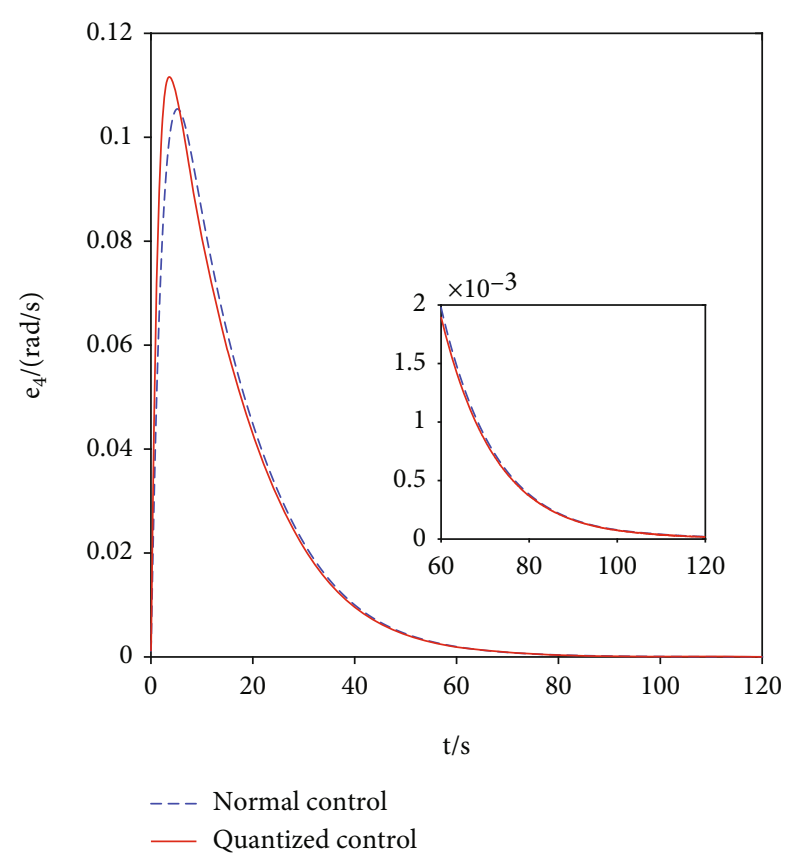

FIGURE 21: Relative angular velocity error curves.

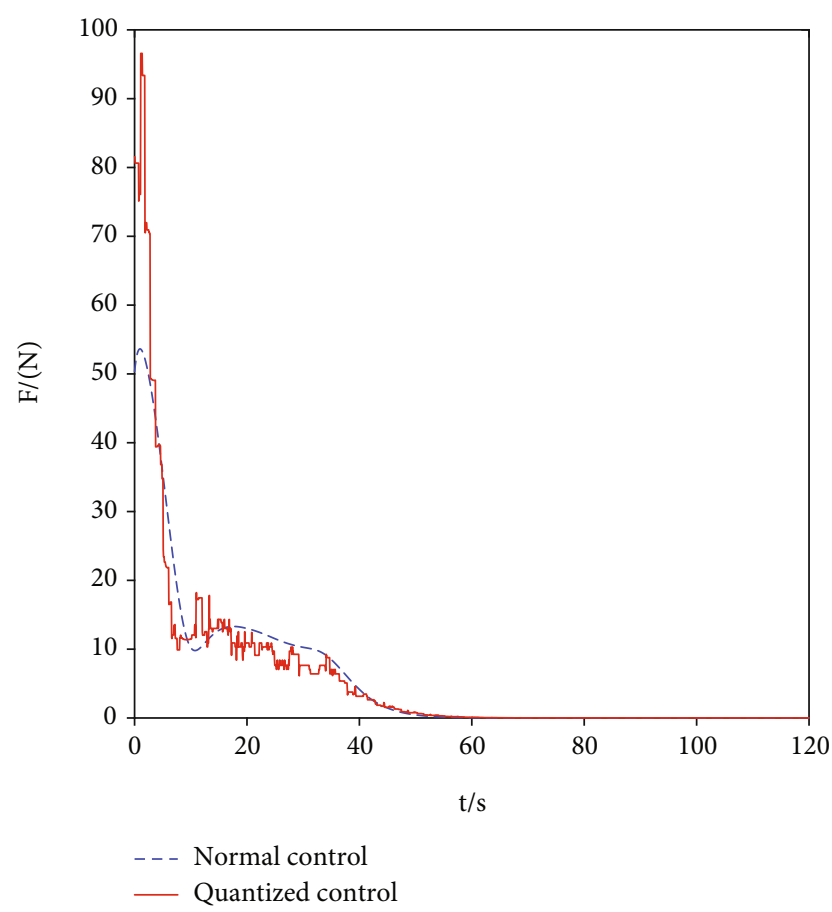

FIgURe 22: Control force curves.

tracking error converges to less than $10^{-3} \times\left[\begin{array}{lll}5 & 5 & 8\end{array}\right]^{\mathrm{T}} \mathrm{m} / \mathrm{s}$ in $53 \mathrm{~s}$. In Figure 6, the curves of $i=1,2,3$ denote the feedback control torque and curves of $i=4,5,6$ denote the feedback control force. After $70 \mathrm{~s}$, the change of control force in a small scale exists because of the existence of a disturbance, especially the time-varying disturbance $\varphi^{d}$. Figure 7 implies that the estimated value of $D$ is stable.

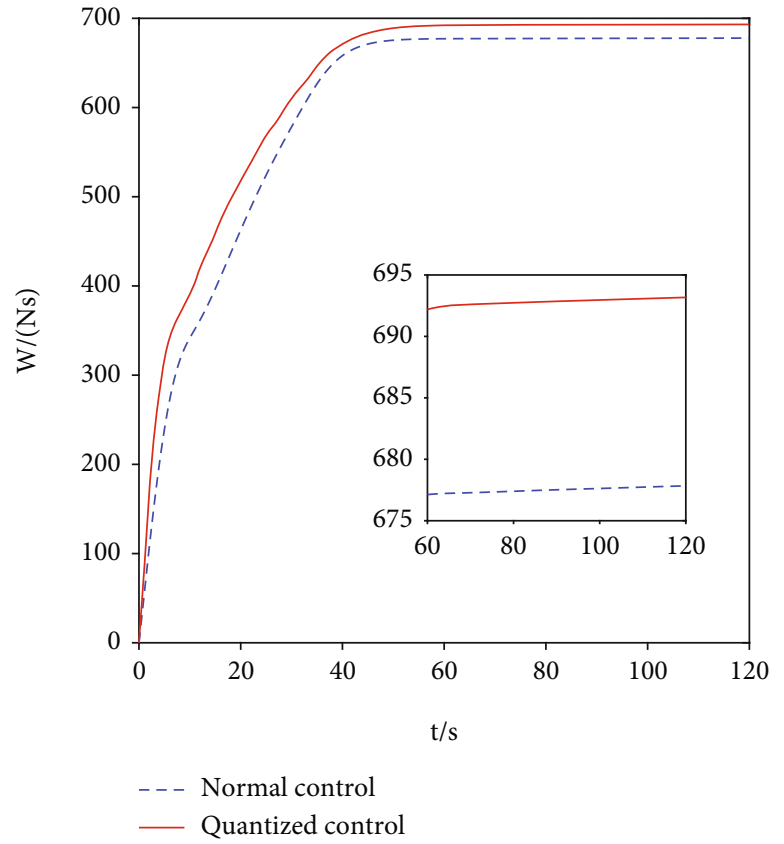

Figure 23: Curves of integration of control force.

Figures 4-7 indicate that the proposed control algorithm is effective, which can provide the system with high performance and stability. The contrastive results are shown in Figures 8-13.

To better show the contrastive results, several notations are defined. $F=\operatorname{norm}(u)$ represents the control force, and $W=\sum F \mathrm{dt}$ is the integration of $F . e_{i}(i=1,2,3,4)$ is defined to express the tracking error of system states. In detail, $e_{1}$ $=\operatorname{norm}(\tilde{b})$ with $\tilde{b}=S(\Theta) \tilde{\beta}, e_{2}=(\widetilde{\theta} / \pi) \times 180$ with $\tilde{\theta}=$ norm $(\tilde{\Theta}), e_{3}=\operatorname{norm}(\tilde{v})$ with $\tilde{v}=\left[\begin{array}{lll}\tilde{\eta}(4) & \tilde{\eta}(5) & \tilde{\eta}(6)\end{array}\right]^{\mathrm{T}}$, and $e_{4}=$ $\operatorname{norm}(\tilde{\Omega})$ with $\tilde{\Omega}=\left[\begin{array}{lll}\tilde{\eta}(1) & \tilde{\eta}(2) & \tilde{\eta}(3)\end{array}\right]^{\mathrm{T}}$.

According to Figure 9, the power consumptions of three control schemes are approximately equal after $60 \mathrm{~s}$. Consequently, the comparative simulations satisfy the premise of equal power consumption. Figures 10-13 indicates that the convergence performances are roughly the same on the whole. More details about the stability can be found in Table 6 .

More information can be found in Figures 10-13: (1) the chattering phenomenon is obvious in $S_{\text {sig }}$. (2) The final convergence region of $S_{\text {tanh }}$ is bigger than that of $S_{\text {sig }}$ and $S_{\text {proposed }}$, which means that the control accuracy of $S_{\text {tanh }}$ is lower. Take Figure 10 for example, the position tracking error under $S_{\text {sig }}$ converges to less than $1 \times 10^{-7} \mathrm{~m}$ in $90 \mathrm{~s}$, but with a severely chattering, which can be seen in Figure $10(\mathrm{c})$. The position tracking error under $S_{\text {tanh }}$ converges to less than $1.6 \times 10^{-3} \mathrm{~m}$ in $90 \mathrm{~s}$ while that of $S_{\text {proposed }}$ converges to less than $1.6 \times 10^{-4} \mathrm{~m}$ in $90 \mathrm{~s}$ (see Figures 10(a) and 10(b)). As a result, two prominent advantages of $S_{\text {proposed }}$ can be found by means of the steady-state analysis. Firstly, in comparison to $S_{\text {sig }}$, the chattering problem is reduced by virtue of the chattering-free control input 

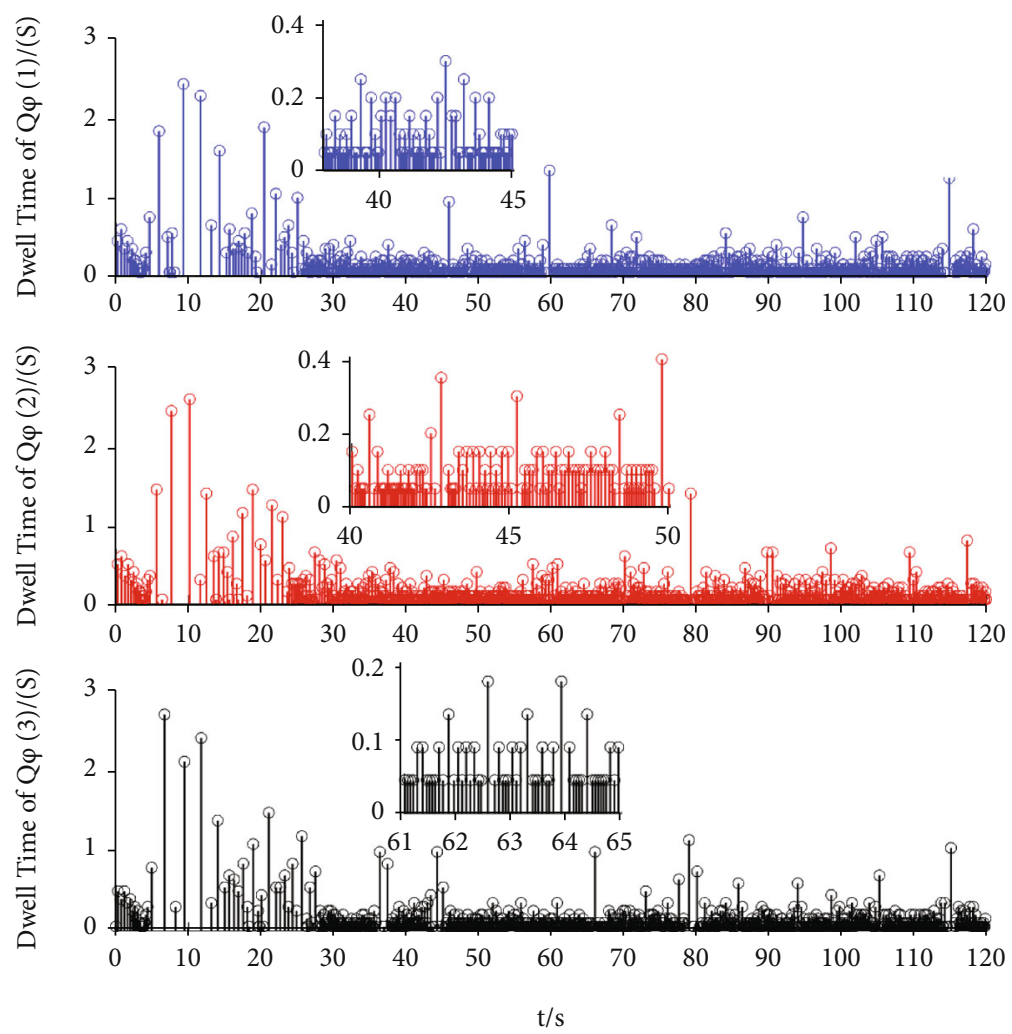

Figure 24: The dwell time of control torque.
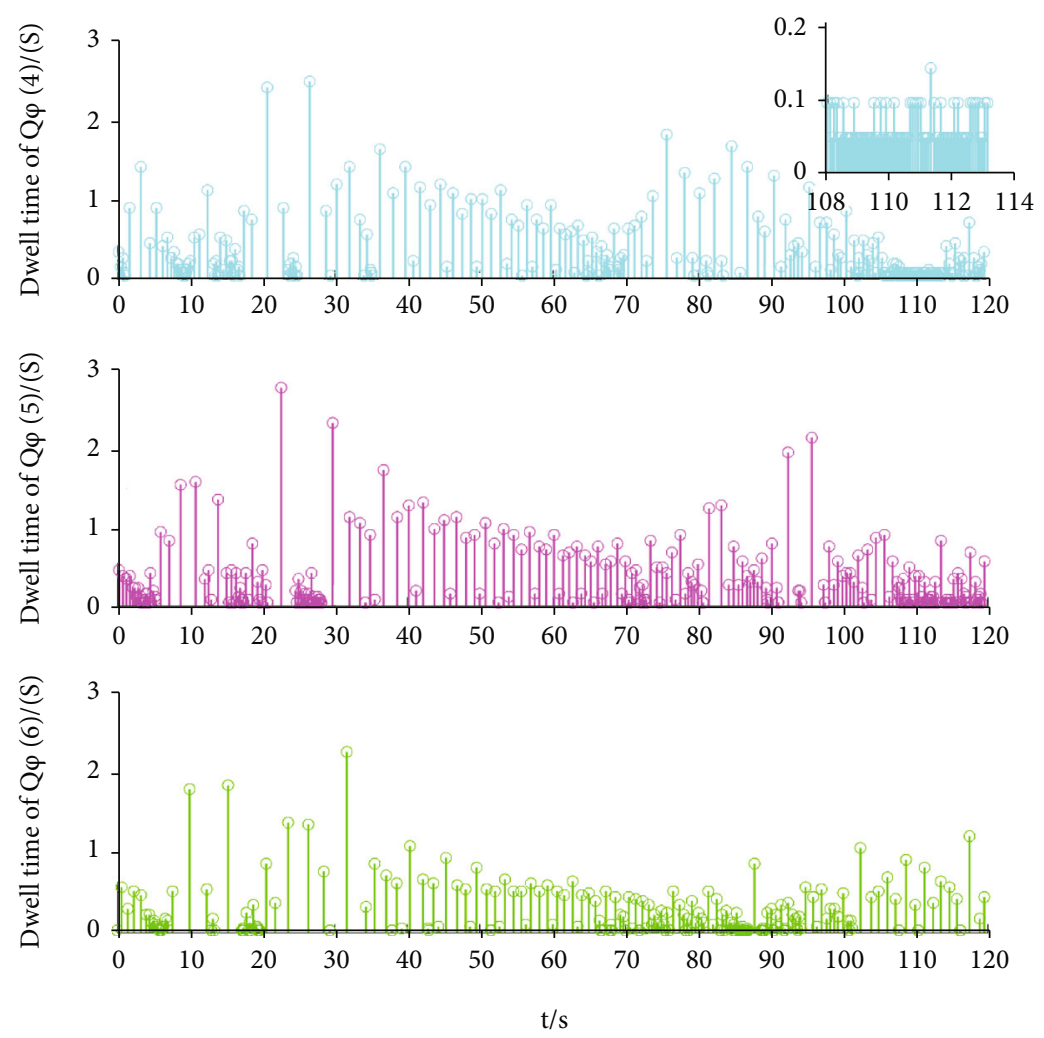

FIGURE 25: The dwell time of control force. 
in $S_{\text {proposed }}$. Another merit is that $S_{\text {proposed }}$ has a better error tracking accuracy than $S_{\text {tanh }}$.

Two conclusions can be drawn through the above results: (1) the proposed method can guarantee the system with finite-time stability and has a better performance of system robustness and control accuracy. (2) The chattering phenomenon in SMC can be effectively reduced by the proposed method.

4.2. Simulation Results of Adaptive Quantized TSMC Scheme. An HLQ is adopted in this paper to validate the feasibility of the proposed quantized TSMC scheme. The quantizer parameters are chosen to be $u=0.0008$ and $\delta=0.33$. The SMS and control inputs of $S_{\text {proposed }}$ is implemented here but with no change in SMS gains and control gains in Table 5. Simulation results of the quantized control are presented in Figures 14-17. Figures 18-23 show the comparisons between normal control and quantized control.

Compared with Figures 3 and 4, similar simulation results are obtained in Figures 14 and 15, which implies that quantized control is effective for error tracking.

As the designed continuous control inputs in Equation (39) are quantized, the change of control forces is discontinuous in Figure 16. Figures 24 and 25 show the dwell times of quantized control torque and force, from which it concludes that data transmission frequency is considerably reduced.

Thus, the HLQ implemented here is feasible and effective for the coupled relative motions control of spacecraft RVD. More contrastive results are shown in Figures 18-23.

Figures 22 and 23 indicate that a small increment comes to the control force of the quantized control scheme. Though the convergence rate is faster than normal control without quantization seemingly, there is a small delay (about 2-5s) in convergence time of quantized control, which can be seen in Figures 18-21. Apart from the improvement of convergence rate, the tiny increase of power consumption leads to better control accuracy. The changes in control accuracy are small, and a distinct result can be seen in Figure 18.

In general, the quantized TSMC scheme can guarantee the error tracking system with high stability and control accuracy, which is similar to that of normal control. Meanwhile, the information transmission is reduced by means of an HLQ.

\section{Conclusion}

This paper presents a finite-time control scheme on SE(3) for the final proximity maneuver of spacecraft RVD subject to external disturbance and input quantization. By feat of Lie group SE(3), the integrated model of translational and rotational motion can simplify the controller design and avoid coupling. The proposed TSMC algorithm can drive the states of the error tracking system to the equilibrium points in finite-time, which is proved rigorously with no knowledge of the upper bound values of adaptive parameters. The frequency of information transmission is effectively reduced by the quantized controller. Meanwhile, the pro- posed control algorithm possesses a superiority of better control precision, reduced chattering, and nonsingularity. Finally, simulation results demonstrate the effectiveness of the proposed scheme.

5.1. Future Work. Besides these advantages, there are two drawbacks in this paper. Firstly, the presented control scheme requires a precise model of the chaser, which may be unfeasible in missions with uncertain inertia. Secondly, the quantizer parameters for different dimensions of control torque and force are assumed to be the same. Adaptive methods or neural networks can be referenced to tackle these two limitations in future work.

\section{Appendix}

\section{A. Proof of $\left\|R_{e}\right\|_{2}=1$}

Let initial relative attitude be $\theta(0 \leq \theta \leq 2 \pi)$, the initial relative principal rotation axis is chosen as $\left[\begin{array}{lll}a & b & c\end{array}\right]^{\mathrm{T}}$ with $a$, $b$, and $c$ being constants. The actual values can be seen in Table 2. Consequently, the attitude tracking error $\tilde{\Theta} \in \mathbb{R}^{3}$ in Equation (17) can be obtained as

$$
\tilde{\Theta}=\frac{\theta}{\sqrt{a^{2}+b^{2}+c^{2}}}\left[\begin{array}{lll}
a & b & c
\end{array}\right]^{\mathrm{T}}=\theta\left[\begin{array}{lll}
d & e & f
\end{array}\right]^{\mathrm{T}},
$$

with $d, e$, and $f$ being constants and $d^{2}+e^{2}+f^{2}=1$. Hence, $\tilde{\eta}^{\vee}=\left[\begin{array}{c}\tilde{\Theta} \\ \tilde{\beta}\end{array}\right]^{\vee}=\left[\begin{array}{cc}\tilde{\Theta}^{\vee} & \tilde{\beta} \\ 0_{1 \times 3} & 0\end{array}\right]$ with $\tilde{\Theta}^{\vee}=\theta\left[\begin{array}{ccc}0 & -f & e \\ f & 0 & -d \\ -e & d & 0\end{array}\right]$.

According to $\operatorname{Remark} 2, \quad \exp _{\mathrm{SE}(3)}\left(\left[\begin{array}{c}\tilde{\Theta} \\ \tilde{\beta}\end{array}\right]^{\vee}\right)=h_{e}=$ $\left[\begin{array}{cc}R_{e} & b_{e} \\ 0_{1 \times 3} & 1\end{array}\right]$ is valid. It yields that $\exp _{\mathrm{SE}(3)}\left(\left[\begin{array}{cc}\tilde{\Theta}^{\times} & \tilde{\beta} \\ 0_{1 \times 3} & 0\end{array}\right]\right)=$ $\left[\begin{array}{cc}R_{e} & b_{e} \\ 0_{1 \times 3} & 1\end{array}\right]$. According to [36], one has $R_{e}=I+\sin \|\tilde{\Theta}\|$ $\left(\tilde{\Theta}^{\vee} /\|\tilde{\Theta}\|\right)+(1-\cos \|\tilde{\Theta}\|)\left(\tilde{\Theta}^{\vee} /\|\tilde{\Theta}\|\right)^{2}$. By virtue of the expression of $\tilde{\Theta}$ in (A.1), it has

$$
\begin{aligned}
R_{e}= & I+\frac{\sin \theta}{\theta} \tilde{\Theta}^{v}+\frac{(1-\cos \theta)}{\theta^{2}}\left(\tilde{\Theta}^{v}\right)^{2} \\
& =I+\sin \theta\left[\begin{array}{ccc}
0 & -f & e \\
f & 0 & -d \\
-e & d & 0
\end{array}\right]+(1-\cos \theta)\left[\begin{array}{ccc}
0 & -f & e \\
f & 0 & -d \\
-e & d & 0
\end{array}\right] \\
= & I+\sin \theta\left[\begin{array}{ccc}
0 & -f & e \\
f & 0 & -d \\
-e & d & 0
\end{array}\right]+(1-\cos \theta)\left[\begin{array}{ccc}
-e^{2}-f^{2} & d e & d f \\
d e & -d^{2}-f^{2} & e f \\
d f & e f & -d^{2}-e^{2}
\end{array}\right] \\
& {\left[\begin{array}{ccc}
d^{2}+\left(e^{2}+f^{2}\right) \cos \theta & d e(1-\cos \theta)-f \sin \theta & d f(1-\cos \theta)+e \sin \theta \\
d e(1-\cos \theta)+f \sin \theta & e^{2}+\left(d^{2}+f^{2}\right) \cos \theta & e f(1-\cos \theta)-d \sin \theta \\
d f(1-\cos \theta)-e \sin \theta & e f(1-\cos \theta)+d \sin \theta & f^{2}+\left(d^{2}+e^{2}\right) \cos \theta
\end{array}\right] . }
\end{aligned}
$$

The conjugate transpose matrix of $R_{e}$ is 


$$
R_{e}^{\mathrm{H}}=\left[\begin{array}{ccc}
d^{2}+\left(e^{2}+f^{2}\right) \cos \theta & d e(1-\cos \theta)+f \sin \theta & d f(1-\cos \theta)-e \sin \theta \\
d e(1-\cos \theta)-f \sin \theta & e^{2}+\left(d^{2}+f^{2}\right) \cos \theta & e f(1-\cos \theta)+d \sin \theta \\
d f(1-\cos \theta)+e \sin \theta & e f(1-\cos \theta)-d \sin \theta & f^{2}+\left(d^{2}+e^{2}\right) \cos \theta
\end{array}\right] .
$$

Then it concludes that $R_{e}^{\mathrm{H}} R_{e}=R_{e} R_{e}^{\mathrm{H}}=I$. Hence, $R_{e}$ is a unitary matrix. According to the definition of spectral norm, $\left\|R_{e}\right\|_{2}=\rho\left(R_{e}^{\mathrm{H}} R_{e}\right)^{1 / 2}=1$. This concludes the proof.

\section{B. Proof of Lemma 7}

For a vector $\mathbf{x}=\left[\begin{array}{llll}x_{1} & x_{2} & \cdots & x_{n}\end{array}\right]^{\mathrm{T}}$, it is clear that $x^{\mathrm{T}} x^{2 \alpha+1}$ $=\sum_{I-1}^{N}\left|x_{i}\right|^{2(\alpha+1)}$. According to Lemma 6, one has

$$
\left(\left|x_{1}\right|^{2}+\cdots+\left|x_{n}\right|^{2}\right)^{(\alpha+1)} \leq \sum_{i=1}^{n}\left|x_{i}\right|^{2(\alpha+1)}=x^{\mathrm{T}} x^{2 \alpha+1} .
$$

And for any $\varepsilon>0$, one has

$$
\sqrt{\left(\left|x_{1}\right|^{2}+\cdots+\left|x_{n}\right|^{2}\right)^{(\alpha+1)}+\varepsilon^{2}} \leq\left(\left|x_{1}\right|^{2}+\cdots+\left|x_{n}\right|^{2}\right)^{(\alpha+1) / 2}+\varepsilon
$$

Therefore,

$$
\frac{\left(\left|x_{1}\right|^{2}+\cdots+\left|x_{n}\right|^{2}\right)^{(\alpha+1)}}{\left(\left|x_{1}\right|^{2}+\cdots+\left|x_{n}\right|^{2}\right)^{(\alpha+1) / 2}+\varepsilon} \leq \frac{\sum_{i=1}^{n}\left|x_{i}\right|^{2(\alpha+1)}}{\sqrt{\left(\left|x_{1}\right|^{2}+\cdots+\left|x_{n}\right|^{2}\right)^{(\alpha+1)}+\varepsilon^{2}}} .
$$

Then, it yields

$$
\begin{aligned}
\left(\sum_{i=1}^{n} x_{i}^{2}\right)^{(\alpha+1) / 2}- & \frac{\sum_{i=1}^{n}\left|x_{i}\right|^{2(\alpha+1)}}{\sqrt{\left(\sum_{i=1}^{n}\left|x_{i}\right|^{2}\right)^{(\alpha+1)}+\varepsilon^{2}}} \leq\left(\sum_{i=1}^{n} x_{i}^{2}\right)^{(\alpha+1) / 2} \\
& -\frac{\left(\sum_{i=1}^{n}\left|x_{i}\right|^{2}\right)^{(\alpha+1)}}{\left(\sum_{i=1}^{n}\left|x_{i}\right|^{2}\right)^{(\alpha+1) / 2}+\varepsilon}<\varepsilon .
\end{aligned}
$$

It is known that $\|x\|^{2}=\sum_{i=1}^{n}\left|x_{i}\right|^{2}$. Therefore, $-\left(x^{\mathrm{T}} x^{2 \alpha+1}\right.$ / $\sqrt{\left.\|x\|^{2(\alpha+1)}+\varepsilon^{2}\right)} \leq-\|x\|^{\alpha+1}+\varepsilon$ in Equation (29) is proven.

\section{Data Availability}

No data were used to support this study.

\section{Conflicts of Interest}

The authors declare that there are no conflicts of interest regarding publication of this article.

\section{Acknowledgments}

The authors acknowledge financial support from the National Natural Science Foundation of China (No. 11902347). Thanks are due to Dr. Huang Bing from Science and Technology on Underwater Vehicle Laboratory of Harbin Engineering University, for his technical support on this article.

\section{References}

[1] K. Gong, Y. Liao, and Y. Wang, "Adaptive fixed-time terminal sliding mode control on $\mathrm{SE}(3)$ for coupled spacecraft tracking maneuver," International Journal of Aerospace Engineering, vol. 2020, Article ID 3085495, 15 pages, 2020.

[2] J. Zhang, J. Biggs, D. Ye, and Z. Sun, "Extended-state-observerbased event-triggered orbit-attitude tracking for low-thrust spacecraft," IEEE Transactions on Aerospace and Electronic Systems, vol. 56, no. 4, pp. 2872-2883, 2019.

[3] Y. Wang, H. Hong, J. Guo, X. Wang, and W. Shang, "Configuration error function design and application to fixed-time geometric terminal sliding-mode control on SE(3)," Acta Astronautica, vol. 174, pp. 61-71, 2020.

[4] D. Lee, G. Vukovich, and H. Gui, "Adaptive variable-structure finite-time mode control for spacecraft proximity operations with actuator saturation," Advances in Space Research, vol. 59, no. 10, pp. 2473-2487, 2017.

[5] Z. Shi, C. Deng, S. Zhang, Y. Xie, H. Cui, and Y. Hao, "Hyperbolic tangent function-based finite-time sliding mode control for spacecraft rendezvous maneuver without chattering," IEEE Access, vol. 8, pp. 60838-60849, 2020.

[6] Y. Liu, G. Tang, Y. Li, H. Li, J. Ren, and S. Zhang, "Adaptive finite-time control for spacecraft rendezvous under unknown system parameters," International Journal of Aerospace Engineering, vol. 2020, Article ID 3648260, 13 pages, 2020.

[7] C. Tan, G. Xu, L. Dong, H. Zhao, J. Li, and S. Zhang, "Neural network-based finite-time fault-tolerant control for spacecraft without unwinding," International Journal of Aerospace Engineering, vol. 2021, Article ID 9269438, 10 pages, 2021.

[8] J. Wu, K. Liu, and D. Han, "Adaptive sliding mode control for six-DOF relative motion of spacecraft with input constraint," Acta Astronautica, vol. 87, pp. 64-76, 2013.

[9] H. Gui and G. Vukovich, "Dual-quaternion-based adaptive motion tracking of spacecraft with reduced control effort," Nonlinear Dynamics, vol. 83, no. 1-2, pp. 597-614, 2016. 
[10] X. Wang, D. Han, C. Yu, and Z. Zheng, "The geometric structure of unit dual quaternion with application in kinematic control," Journal of Mathematical Analysis and Applications, vol. 389, no. 2, pp. 1352-1364, 2012.

[11] L. Sun and H. Wei, "6-DOF integrated adaptive backstepping control for spacecraft proximity operations," IEEE Transactions on Aerospace and Electronic Systems, vol. 51, no. 3, pp. 2433-2443, 2015.

[12] L. Sun, W. Huo, and Z. Jiao, "Adaptive nonlinear robust relative pose control of spacecraft autonomous rendezvous and proximity operations," ISA Transactions, vol. 67, pp. 47-55, 2017.

[13] L. Sun, W. Huo, and Z. Jiao, "Adaptive backstepping control of spacecraft rendezvous and proximity operations with input saturation and full-state constraint," IEEE Transactions on Industrial Electronics, vol. 64, no. 1, pp. 480-492, 2016.

[14] J. Biggs and L. Colley, "Geometric attitude motion planning for spacecraft with pointing and actuator constraints," Journal of Guidance, Control, and Dynamics, vol. 39, no. 7, pp. 16721677, 2016.

[15] D. Lee and G. Vukovich, "Robust adaptive terminal sliding mode control on SE(3) for autonomous spacecraft rendezvous and docking," Nonlinear Dynamics, vol. 83, no. 4, pp. 22632279, 2016.

[16] J. Zhang, D. Ye, Z. Sun, and C. Liu, "Extended state observer based robust adaptive control on SE(3) for coupled spacecraft tracking maneuver with actuator saturation and misalignment," Acta Astronautica, vol. 143, pp. 221-233, 2018.

[17] A. Müller and Z. Terze, "The significance of the configuration space Lie group for the constraint satisfaction in numerical time integration of multibody systems," Mechanism and Machine Theory, vol. 82, pp. 173-202, 2014.

[18] A. Müller, "Screw and Lie group theory in multibody kinematics," Multibody System Dynamics, vol. 43, no. 1, pp. 37-70, 2018.

[19] K. Yang, W. Yang, and C. Wang, "Inverse dynamic analysis and position error evaluation of the heavy-duty industrial robot with elastic joints: an efficient approach based on Lie group," Nonlinear Dynamics, vol. 93, no. 2, pp. 487-504, 2018.

[20] H. Henninger, K. D. von Ellenrieder, and J. Biggs, “Trajectory generation and tracking on SE(3) for an underactuated AUV with disturbances," IFAC-Papers OnLine, vol. 52, no. 21, pp. 242-247, 2019.

[21] D. Lee, A. K. Sanyal, and E. A. Butcher, “Asymptotic tracking control for spacecraft formation flying with decentralized collision avoidance," Journal of Guidance, Control, and Dynamics, vol. 38, no. 4, pp. 587-600, 2015.

[22] J. Zhang, D. Ye, J. D. Biggs, and Z. Sun, "Finite-time relative orbit-attitude tracking control for multi-spacecraft with collision avoidance and changing network topologies," Advances in Space Research, vol. 63, no. 3, pp. 1161-1175, 2019.

[23] A. Sanyal and N. Nordkvist, "Attitude state estimation with multirate measurements for almost global attitude feedback tracking," Journal of Guidance, Control, and Dynamics, vol. 35, no. 3, pp. 868-880, 2012.

[24] M. Nazari, E. A. Butcher, T. Yucelen, and A. K. Sanyal, "Decentralized consensus control of a rigid-body spacecraft formation with communication delay," Journal of Guidance, Control, and Dynamics, vol. 39, no. 4, pp. 838-851, 2016.

[25] K. Phogat, D. Chatterjee, and R. Banavar, "Discrete-time optimal attitude control of a spacecraft with momentum and con- trol constraints," Journal of Guidance, Control, and Dynamics, vol. 41, no. 1, pp. 199-211, 2018.

[26] Y. Guo, B. Huang, J. Guo, A. Li, and C. Wang, "Velocity-free sliding mode control for spacecraft with input saturation," Acta Astronautica, vol. 154, pp. 1-8, 2019.

[27] G. Vazquez, M. Saleh, and A. Leonardo, "Robust control design to the furuta system under time delay measurement feedback and exogenous-based perturbation," Mathematics, vol. 8, no. 12, p. 2131, 2020.

[28] S. Rasoolinasab, S. Mobayen, A. Fekih, P. Narayan, and Y. Yao, "A composite feedback approach to stabilize nonholonomic systems with time varying time delays and nonlinear disturbances," ISA Transactions, vol. 101, pp. 177-188, 2020.

[29] C. Zhang, G. Ma, Y. Sun, and C. Li, "Simple model-free attitude control design for flexible spacecraft with prescribed performance," Proceedings of the Institution of Mechanical Engineers, Part G: Journal of Aerospace Engineering, vol. 233, no. 8, pp. 2760-2771, 2019.

[30] S. Venkataraman and S. Gulati, "Terminal sliding modes: a new approach to nonlinear control synthesis," in Fifth International Conference on Advanced Robotics 'Robots in Unstructured Environments, vol. 1, pp. 443-448, Pisa, Italy, 1991.

[31] B. Huang, A. Li, Y. Guo, and C. Wang, "Finite-time faulttolerant attitude tracking control for spacecraft without unwinding," Proceedings of the Institution of Mechanical Engineers, Part G: Journal of Aerospace Engineering, vol. 233, no. 6, pp. 2119-2130, 2019.

[32] Y. Guo, B. Huang, S. Wang, A. Li, and C. Q. Wang, “Adaptive finite-time control for attitude tracking of spacecraft under input saturation," Journal of Aerospace Engineering, vol. 31, no. 2, article 04017086, 2018.

[33] B. Wu, "Spacecraft attitude control with input quantization," Journal of Guidance, Control, and Dynamics, vol. 39, no. 1, pp. 176-181, 2016.

[34] B. Huang, B. Zhou, S. Zhang, and C. Zhu, "Adaptive prescribed performance tracking control for underactuated autonomous underwater vehicles with input quantization," Ocean Engineering, vol. 221, p. 108549, 2021.

[35] L. Su and G. Chesi, "Robust stability of uncertain linear systems with input and output quantization and packet loss," Automatica, vol. 87, pp. 267-273, 2018.

[36] Y. Shi, X. Shao, and W. Zhang, "Quantized learning control for flexible air-breathing hypersonic vehicle with limited actuator bandwidth and prescribed performance," Aerospace Science and Technology, vol. 97, p. 105629, 2020.

[37] J. Zhou, C. Wen, and W. Wang, "Adaptive control of uncertain nonlinear systems with quantized input signal," Automatica, vol. 95, pp. 152-162, 2018.

[38] S. Esmaeilzadeh, G. Mehdi, and M. Saleh, "Chattering-free fault-tolerant attitude control with fast fixed-time convergence for flexible spacecraft," International Journal of Control, Automation and Systems, vol. 19, no. 2, pp. 767-776, 2021.

[39] Q. Li, C. Sun, S. Song, Q. Gou, and Z. Niu, Robust Adaptive Control for Spacecraft Final Proximity Maneuvers with Safety Constraint and Input Quantization, ISA transactions, 2021.

[40] F. Bullo and R. Murray, "Proportional derivative (PD) control on the Euclidean group," in European Control Conference Citeseer, pp. 1091-1097, Zurich, Switzerland, September 1995.

[41] X. Shao and Y. Shi, "Neural adaptive control for MEMS gyroscope with full-state constraints and quantized input," IEEE 
Transactions on Industrial Informatics, vol. 16, no. 10, pp. 6444-6454, 2020.

[42] Q. Hu, B. Jiang, and M. Friswell, "Robust saturated finite time output feedback attitude stabilization for rigid spacecraft," Journal of Guidance, Control, and Dynamics, vol. 37, no. 6, pp. 1914-1929, 2014.

[43] Y. Mei, Y. Liao, K. Gong, and D. Luo, "Adaptive fuzzy modified fixed-time fault-tolerant control on SE (3) for coupled spacecraft," Mathematical Problems in Engineering, vol. 2021, Article ID 6648578, 21 pages, 2021. 